\title{
Generation of new fractional inequalities via $n$ polynomials $s$-type convexity with applications
}

\author{
Saima Rashid ${ }^{1}$, Imdat Işcan², Dumitru Baleanu ${ }^{3}$ and Yu-Ming Chu ${ }^{4,5^{*}}$
}

"Correspondence:

chuyuming2005@126.com

${ }^{4}$ Department of Mathematics,

Huzhou University, Huzhou, China

${ }^{5}$ Hunan Provincial Key Laboratory of

Mathematical Modeling and

Analysis in Engineering, Changsha

University of Science \& Technology,

Changsha, China

Full list of author information is

available at the end of the article

\section{Springer}

\begin{abstract}
The celebrated Hermite-Hadamard and Ostrowski type inequalities have been studied extensively since they have been established. We find novel versions of the Hermite-Hadamard and Ostrowski type inequalities for the $n$-polynomial s-type convex functions in the frame of fractional calculus. Taking into account the new concept, we derive some generalizations that capture novel results under investigation. We present two different general techniques, for the functions whose first and second derivatives in absolute value at certain powers are $n$-polynomial $s$-type convex functions by employing $\mathcal{K}$-fractional integral operators have yielded intriguing results. Applications and motivations of presented results are briefly discussed that generate novel variants related to quadrature rules that will be helpful for in-depth investigation in fractal theory, optimization and machine learning.
\end{abstract}

MSC: 26D15; 26D10; 90C23; 26E60

Keywords: Convex function; s-type convex function; Hermite-Hadamard inequality; Ostrowski inequality; Higher degree polynomial s-convex

\section{Introduction and preliminaries}

A few decades ago, the classical calculus has been revolutionized by tremendous innovations. The researchers are concurring with the extraordinary excellence and truthfulness in outcomes by the fractional-order equations. If we observed the historical background of fractional calculus, the notion has been instigated from a letter of L'Hospital to Leibniz for the meaning of $d r / d r^{n}$ for $n=1 / 2$. In response, Leibniz saw this is "An obvious conundrum, from which one day valuable outcomes will be shown". Also, presently one witnesses the utilization of fractional calculus in various areas, for example, chaos, simulation, and modeling. Numerous useful definitions and operators show the beauty of the fractional calculus, for instance, the Riemann, Caputo, Hadamard, Katugampola, Erdelyi-Kober, Atangana-Baleanu, Weyl types and many others with potential applications in mathematics and physics [1-11]. For a feature depiction of the origin of fractional calculus, improvements, and applications, we refer the reader to the notable monographs $[12,13]$ and the interesting articles [14-25].

(c) The Author(s) 2020. This article is licensed under a Creative Commons Attribution 4.0 International License, which permits use, sharing, adaptation, distribution and reproduction in any medium or format, as long as you give appropriate credit to the original author(s) and the source, provide a link to the Creative Commons licence, and indicate if changes were made. The images or other third party material in this article are included in the article's Creative Commons licence, unless indicated otherwise in a credit line to the material. If material is not included in the article's Creative Commons licence and your intended use is not permitted by statutory regulation or exceeds the permitted use, you will need to obtain permission directly from the copyright holder. To view a copy of this licence, visit http://creativecommons.org/licenses/by/4.0/. 
Convexity has played a crucial role in the advancement of pure and applied mathematics [26-35]. Due to its robustness, convex functions and convex sets have been generalized and extended in many mathematics branches, in particular, many inequalities can be found in the literature [36-48] via convexity theory. To the best of our knowledge, the Hermite-Hadamard inequality is a well-known, paramount and extensively useful inequality in the applied literature of mathematical inequalities [49-60]. This inequality is of pivotal significance because of other classical inequalities such as the Hardy, Opial, Lynger, Ostrowski, Minkowski, Hölder, Ky-Fan, Beckenbach-Dresher, Levinson, arithmeticgeometric, Young, Olsen and Gagliardo-Nirenberg inequalities, which are closely related to the classical Hermite-Hadamard inequality [61]. It can be stated as follows: the double inequality

$$
\mathcal{P}\left(\frac{\eta_{1}+\eta_{2}}{2}\right) \leq \frac{1}{\eta_{2}-\eta_{1}} \int_{\eta_{1}}^{\eta_{2}} \mathcal{P}(r) d r \leq \frac{\mathcal{P}\left(\eta_{1}\right)+\mathcal{P}\left(\eta_{2}\right)}{2}
$$

holds if $\mathcal{P}$ is a convex function on the interval $\left[\eta_{1}, \eta_{2}\right]$.

Recently, the Hermite-Hadamard inequality (1.1) and its generalizations, refinements, extensions and variants have attracted the attention of many researchers. It has been proved that the function $\mathcal{P}: I \rightarrow \mathbb{R}$ is convex if and only if inequality (1.1) holds for all $\eta_{1}, \eta_{2} \in I$ with $\eta_{1} \neq \eta_{2}$.

Let $\mathcal{I} \subseteq \mathbb{R}$ and $\mathcal{P}: \mathcal{I} \rightarrow \mathbb{R}$ be a differentiable mapping on $\mathcal{I}^{\circ}$ (the interior of $\mathcal{I}$ ) such that $\eta_{1}, \eta_{2} \in \mathcal{I}^{\circ}$ with $\eta_{1}<\eta_{2}$. Then the well-known Ostrowski inequality [62] states that

$$
\left|\mathcal{P}(z)-\frac{1}{\eta_{2}-\eta_{1}} \int_{\eta_{1}}^{\eta_{2}} \mathcal{P}(z) d z\right| \leq\left[\frac{1}{4}+\frac{\left(z-\frac{\eta_{1}+\eta_{2}}{2}\right)^{2}}{\left(\eta_{2}-\eta_{1}\right)^{2}}\right]\left(\eta_{2}-\eta_{1}\right) \mathcal{M}
$$

for all $z \in\left[\eta_{1}, \eta_{2}\right]$ if $\left|\mathcal{P}^{\prime}(\zeta)\right| \leq \mathcal{M}$ for all $\zeta \in\left[\eta_{1}, \eta_{2}\right]$.

Ostrowski type inequalities have significant contributions in the numerical analysis as they provide the error estimates of many quadrature rules. In recent years, they have been extended and generalized in many fields.

The uses of variants in applied sciences are generally studied and now it is a profoundly appealing research-oriented area where the researchers also investigate the existence and uniqueness of the solutions of fractional differential equations. Khan et al. [63] derived the Hermite-Hadamard inequality for $s$-convex functions. In [64], the authors derived several generalizations for the Ostrowski type inequality involving the generalized $\mathcal{K}$-fractional integrals.

In the article, we propose an novel class of functional variants for convex functions and several other new and effectively applicable generalizations for convexity theory and fractional operators. The novel technique is useful to generate the Mandelbrot and Julia sets for quadratic and cubic polynomials with $s$-convexity [65-67].

Now, we discuss some connections between the class of convex functions and $s$-convex functions.

Definition 1.1 Let $s \in[0,1]$. Then the real-valued function $\mathcal{P}: \mathcal{I} \rightarrow \mathbb{R}$ is said to be $s$-type convex on $\mathcal{I}$ if the inequality

$$
\mathcal{P}(\zeta x+(1-\zeta) y) \leq[1-s(1-\zeta)] \mathcal{P}(x)+(1-s \zeta) \mathcal{P}(y)
$$

holds for all $x, y \in \mathcal{I}$ and $\zeta \in[0,1]$. 
Remark 1.2 From Definition 1.1 we clearly see that:

(1) If we choose $s=1$, then we get the classical convex function.

(2) If we choose $s=0$, then we get the definition of $P$-function in [68].

(3) If $\mathcal{P}$ is $s$-type convex on $\mathcal{I}$, then the range of the function $\mathcal{P}$ is $[0, \infty)$.

Indeed, let $x \in \mathcal{I}$. Then by the $s$-type convexity of $\mathcal{P}$ we have

$$
\mathcal{P}\left(\zeta \eta_{1}+(1-\zeta) x\right) \leq[1-s(1-\zeta)] \mathcal{P}\left(\eta_{1}\right)+(1-s \zeta) \mathcal{P}(x)
$$

for all $\eta_{1} \in \mathcal{I}$ and $\zeta \in[0,1]$.

If $\zeta=1$, then we clearly see that

$$
\mathcal{P}\left(\eta_{1}\right) \leq \mathcal{P}\left(\eta_{1}\right)+(1-s) \mathcal{P}(x),
$$

which leads to the conclusion that $\mathcal{P}(x) \geq 0$.

Proposition 1.3 Every nonegative convex function is also an s-type convex function.

Proof Proposition 1.3 follows easily from the facts that

$$
s(1-\zeta) \leq(1-\zeta), \quad \zeta \geq s \zeta
$$

for all $\zeta \in[0,1]$ and $s \in[0,1]$.

Next, we introduce the definition of $n$-polynomial $s$-type convex function.

Definition 1.4 Let $s \in[0,1]$ and $n \in \mathbb{N}$. Then the real-valued function $\mathcal{P}: \mathcal{I} \rightarrow \mathbb{R}$ is said to be a $n$-polynomial $s$-type convex function if the inequality

$$
\mathcal{P}(\zeta x+(1-\zeta) y) \leq \frac{1}{n} \sum_{i=1}^{n}\left[1-(s(1-\zeta))^{i}\right] \mathcal{P}(x)+\frac{1}{n} \sum_{i=1}^{n}\left[1-(s \zeta)^{i}\right] \mathcal{P}(y)
$$

holds for $x, y \in \mathcal{I}$ and $\zeta \in[0,1]$.

Remark 1.5 From Definition 1.4 we clearly see that the following statements are true:

(1) If we choose $s=0$, then we get the $P$-functions in [68].

(2) If we choose $s=1$, then we get Definition 2 in [69].

(3) If we choose $n=s=1$, then we get Definition 1.1.

(4) If $\mathcal{P}$ is a $n$-polynomial $s$-type convex function, then the range of the function $\mathcal{P}$ is $[0, \infty)$.

Remark 1.6 Every nonnegative $n$-polynomial convex function is also a $n$-polynomial $s$ type convex function due to

$$
\frac{1}{n} \sum_{i=1}^{n}\left[1-((1-\zeta))^{i}\right] \leq \frac{1}{n} \sum_{i=1}^{n}\left[1-s(1-\zeta)^{i}\right]
$$


and

$$
\frac{1}{n} \sum_{i=1}^{n}\left(1-\zeta^{i}\right) \leq \frac{1}{n} \sum_{i=1}^{n}\left[1-(s \zeta)^{i}\right]
$$

for all $\zeta \in[0,1], n \in \mathbb{N}$ and $s \in[0,1]$.

We now demonstrate some essential ideas associated with the fractional integral which is mainly due to Mubeen et al. [70].

Let $\alpha, \mathcal{K}>0, \eta_{1}<\eta_{2}$ and $\mathcal{P} \in L_{1}\left(\left[\eta_{1}, \eta_{2}\right]\right)$. Then the $\mathcal{K}$-fractional integrals of order $\alpha$ are defined by

$$
\mathcal{J}_{\eta_{1}}^{\alpha, \mathcal{K}} \mathcal{P}(r)=\frac{1}{\mathcal{K} \Gamma_{\mathcal{K}}(\alpha)} \int_{\eta_{1}}^{r}(r-\chi)^{\frac{\alpha}{\mathcal{K}}-1} \mathcal{P}(\chi) d \chi \quad(r>\chi)
$$

and

$$
\mathcal{J}_{\eta_{2}}^{\alpha, \mathcal{K}} \mathcal{P}(r)=\frac{1}{\mathcal{K} \Gamma_{\mathcal{K}}(\alpha)} \int_{\eta_{1}}^{r}(\chi-r)^{\frac{\alpha}{\mathcal{K}}-1} \mathcal{P}(\chi) d \chi \quad(r<\chi),
$$

where $\Gamma_{\mathcal{K}}(\alpha)$ is the $\mathcal{K}$-Gamma function [71] defined by

$$
\Gamma_{\mathcal{K}}(\alpha)=\int_{0}^{\infty} \zeta^{\alpha-1} e^{-\frac{\mathcal{\zeta}^{\mathcal{K}}}{\mathcal{K}}} d \zeta
$$

Note that

$$
\Gamma_{\mathcal{K}}(\alpha+\mathcal{K})=\alpha \Gamma_{\mathcal{K}}(\alpha)
$$

and the $\mathcal{K}$-fractional integrals reduce to the RL-fractional integrals if $\mathcal{K}=1$.

Next, we recall the definitions of the Beta function $\mathbb{B}$ and the Gaussian hypergeometric function ${ }_{2} \mathcal{F}_{1}$ :

$$
\mathbb{B}(x, y)=\frac{\Gamma(x) \Gamma(y)}{\Gamma(x+y)}=\int_{0}^{1} \zeta^{x-1}(1-\zeta)^{y-1} d \zeta
$$

and

$$
{ }_{2} \mathcal{F}_{1}(a, b ; c ; z)=\frac{1}{\mathbb{B}(b, c-b)} \int_{0}^{1} \zeta^{b-1}(1-\zeta)^{c-b-1}(1-z \zeta)^{-a} d \zeta
$$

where $\Gamma(x)=\int_{0}^{\infty} t^{x-1} e^{-t} d t$ is the Euler gamma function [72, 73].

The principal purpose of this article is to derive several novel integral inequalities including the Hermite-Hadamard and Ostrowski type inequalities by using $n$-polynomial $s$-type convexity and $\mathcal{K}$-fractional integral operator. By use of the fractional operators, we obtained new estimates for the functions whose first and second derivatives in absolute value at certain powers are $n$-polynomial $s$-type convex functions. Interestingly, the special cases of the presented results are RL-fractional integral inequalities and quadrature rules. Our work's consequences are useful in the generation of fractals using iterative procedures, which is an interesting field of research and has utilities in the improvement of machine learning algorithms. 


\section{Hermite-Hadamard type inequalities for $n$-polynomial s-type convex function}

The aim of this section is to find some inequalities of Hermite-Hadamard type for $n$ polynomial $s$-type convex functions. In what follows, we denote by $L_{1}\left(\left[\eta_{1}, \eta_{2}\right]\right)$ the space of (Lebesgue) integrable functions on the interval $\left[\eta_{1}, \eta_{2}\right]$.

Theorem 2.1 Let $s \in[0,1], \alpha \in(0,1], \mathcal{K}>0, n \in \mathbb{N}, \eta_{2}>\eta_{1}$ and $\mathcal{P}: \mathcal{I}=\left[\eta_{1}, \eta_{2}\right] \rightarrow \mathbb{R}$ be a n-polynomial s-type convex function such that $\mathcal{P} \in L_{1}\left(\left[\eta_{1}, \eta_{2}\right]\right)$. Then one has

$$
\begin{aligned}
& \left(\frac{n(2-s) 2^{n}}{2^{n}(2 n-s(n+1))+s^{n+1}}\right) \mathcal{P}\left(\frac{\eta_{1}+\eta_{2}}{2}\right) \\
& \quad \leq \frac{\Gamma_{\mathcal{K}}(\mathcal{K}+\alpha)}{\left(\eta_{2}-\eta_{1}\right) \mathcal{K}^{\alpha}}\left[\mathcal{J}_{\eta_{1}^{+}}^{\alpha, \mathcal{K}} \mathcal{P}\left(\eta_{2}\right)+\mathcal{J}_{\eta_{2}^{\alpha}}^{\alpha, \mathcal{K}} \mathcal{P}\left(\eta_{1}\right)\right] \\
& \quad \leq \frac{\left[\mathcal{P}\left(\eta_{1}\right)+\mathcal{P}\left(\eta_{2}\right)\right]}{n}\left[\sum_{i=1}^{n}\left[\frac{\alpha\left(2-s^{i}\right)+2 i \mathcal{K}}{\alpha+i \mathcal{K}}-\frac{\alpha s^{i}}{\mathcal{K}} \mathbb{B}\left(\frac{\alpha}{\mathcal{K}}, i+1\right)\right]\right] .
\end{aligned}
$$

Proof Let $z_{1}, z_{2} \in \mathcal{I}$. Then it follows from the $n$-polynomial $s$-type convexity of $\mathcal{P}$ on $\mathcal{I}$ that

$$
\mathcal{P}\left(\frac{z_{1}+z_{2}}{2}\right) \leq \frac{1}{n} \sum_{i=1}^{n}\left[1-\left(\frac{s}{2}\right)^{i}\right]\left[\mathcal{P}\left(z_{1}\right)+\mathcal{P}\left(z_{2}\right)\right]
$$

Let $z_{1}=\zeta \eta_{2}+(1-\zeta) \eta_{1}$ and $z_{2}=\zeta \eta_{1}+(1-\zeta) \eta_{2}$. Then (2.2) leads to

$$
\mathcal{P}\left(\frac{\eta_{1}+\eta_{2}}{2}\right) \leq \frac{1}{n} \sum_{i=1}^{n}\left[1-\left(\frac{s}{2}\right)^{i}\right]\left[\mathcal{P}\left(\zeta \eta_{2}+(1-\zeta) \eta_{1}\right)+\mathcal{P}\left(\zeta \eta_{1}+(1-\zeta) \eta_{2}\right)\right]
$$

Multiplying on both sides of (2.3) by $\zeta^{\frac{\alpha}{K}-1}$ and integrating the obtained inequality with respect to $\zeta$ from 0 to 1 , we get

$$
\begin{aligned}
& \frac{\mathcal{K}}{\alpha}\left(\frac{n(2-s) 2^{n}}{2^{n}(2 n-s(n+1))+s^{n+1}}\right) \mathcal{P}\left(\frac{\eta_{1}+\eta_{2}}{2}\right) \\
& \quad \leq\left[\int_{0}^{1} \zeta \frac{\alpha}{\mathcal{K}^{-1}} \mathcal{P}\left(\zeta \eta_{2}+(1-\zeta) \eta_{1}\right) d \zeta+\int_{0}^{1} \zeta^{\frac{\alpha}{\mathcal{K}}-1} \mathcal{P}\left(\zeta \eta_{1}+(1-\zeta) \eta_{2}\right) d \zeta\right] \\
& \quad \leq \frac{1}{\left(\eta_{2}-\eta_{1}\right)^{\frac{\alpha}{\mathcal{K}}}}\left[\int_{\eta_{1}}^{\eta_{2}}\left(\frac{u-\eta_{1}}{\eta_{2}-\eta_{1}}\right)^{\frac{\alpha}{\mathcal{K}}-1} \mathcal{P}(u) d u+\int_{\eta_{1}}^{\eta_{2}}\left(\frac{\eta_{2}-u}{\eta_{2}-\eta_{1}}\right)^{\frac{\alpha}{\mathcal{K}}-1} \mathcal{P}(u) d u\right] \\
& \quad \leq \frac{\mathcal{K} \Gamma_{\mathcal{K}}(\alpha)}{\left(\eta_{2}-\eta_{1}\right)^{\frac{\alpha}{\mathcal{K}}}}\left[\mathcal{J}_{\eta_{1}^{\alpha}}^{\alpha, \mathcal{K}} \mathcal{P}\left(\eta_{2}\right)+\mathcal{J}_{\eta_{2}^{-}}^{\alpha} \mathcal{K} \mathcal{P}\left(\eta_{1}\right)\right],
\end{aligned}
$$

that is,

$$
\left(\frac{n(2-s) 2^{n}}{2^{n}(2 n-s(n+1))+s^{n+1}}\right) \mathcal{P}\left(\frac{\eta_{1}+\eta_{2}}{2}\right) \leq \frac{\Gamma_{\mathcal{K}}(\mathcal{K}+\alpha)}{\left(\eta_{2}-\eta_{1}\right)^{\alpha}}\left[\mathcal{J}_{\eta_{1}^{+}}^{\alpha, \mathcal{K}} \mathcal{P}\left(\eta_{2}\right)+\mathcal{J}_{\eta_{2}^{-}}^{\alpha, \mathcal{K}} \mathcal{P}\left(\eta_{1}\right)\right]
$$

which gives the proof of the first inequality of (2.1). 
Next, we prove the second inequality of (2.1). Let $\zeta \in[0,1]$. Then from the fact that $\mathcal{P}$ is a $n$-polynomial $s$-type convex function, we get

$$
\mathcal{P}\left(\zeta \eta_{2}+(1-\zeta) \eta_{1}\right) \leq \frac{1}{n} \sum_{i=1}^{n}\left[1-(s \zeta)^{i}\right] \mathcal{P}\left(\eta_{1}\right)+\frac{1}{n} \sum_{i=1}^{n}\left[1-(s(1-\zeta))^{i}\right] \mathcal{P}\left(\eta_{2}\right)
$$

and

$$
\mathcal{P}\left(\zeta \eta_{1}+(1-\zeta) \eta_{2}\right) \leq \frac{1}{n} \sum_{i=1}^{n}\left[1-s \zeta^{i}\right] \mathcal{P}\left(\eta_{2}\right)+\frac{1}{n} \sum_{i=1}^{n}\left[1-(s(1-\zeta))^{i}\right] \mathcal{P}\left(\eta_{1}\right)
$$

Adding the above inequalities gives

$$
\begin{aligned}
& \mathcal{P}\left(\zeta \eta_{2}+(1-\zeta) \eta_{1}\right)+\mathcal{P}\left(\zeta \eta_{1}+(1-\zeta) \eta_{2}\right) \\
& \quad \leq\left[\mathcal{P}\left(\eta_{1}\right)+\mathcal{P}\left(\eta_{2}\right)\right]\left[\frac{1}{n} \sum_{i=1}^{n}[1-s \zeta]^{i}+\frac{1}{n} \sum_{i=1}^{n}\left[1-(s(1-\zeta))^{i}\right]\right] .
\end{aligned}
$$

Multiplying on both sides of (2.4) by $\zeta^{\frac{\alpha}{K}}-1$, integrating the obtained inequality with respect to $\zeta$ from 0 to 1 and then making the change of the variable, we have

$$
\begin{aligned}
& \int_{0}^{1} \zeta^{\frac{\alpha}{K^{-1}}} \mathcal{P}\left(\zeta \eta_{2}+(1-\zeta) \eta_{1}\right) d \zeta+\int_{0}^{1} \zeta^{\frac{\alpha}{\mathcal{K}}-1} \mathcal{P}\left(\zeta \eta_{1}+(1-\zeta) \eta_{2}\right) d \zeta \\
& \quad \leq\left[\mathcal{P}\left(\eta_{1}\right)+\mathcal{P}\left(\eta_{2}\right)\right] \int_{0}^{1} \zeta^{\frac{\alpha}{K}-1}\left[\frac{1}{n} \sum_{i=1}^{n}\left[1-(s \zeta)^{i}\right]+\frac{1}{n} \sum_{i=1}^{n}\left[1-(s(1-\zeta))^{i}\right]\right] d \zeta
\end{aligned}
$$

which leads to the conclusion that

$$
\begin{aligned}
& \frac{\Gamma_{\mathcal{K}}(\mathcal{K}+\alpha)}{\left(\eta_{2}-\eta_{1}\right){ }^{\frac{\alpha}{\mathcal{K}}}}\left[\mathcal{J}_{\eta_{1}^{+}}^{\alpha, \mathcal{K}} \mathcal{P}\left(\eta_{2}\right)+\mathcal{J}_{\eta_{2}^{-}}^{\alpha, \mathcal{K}} \mathcal{P}\left(\eta_{1}\right)\right] \\
& \quad \leq \frac{\left[\mathcal{P}\left(\eta_{1}\right)+\mathcal{P}\left(\eta_{2}\right)\right]}{n}\left[\sum_{i=1}^{n}\left[\frac{\alpha\left(2-s^{i}\right)+2 i \mathcal{K}}{\alpha+i \mathcal{K}}-\frac{\alpha s^{i}}{\mathcal{K}} \mathbb{B}\left(\frac{\alpha}{\mathcal{K}}, i+1\right)\right]\right] .
\end{aligned}
$$

The proof is completed.

Let $s=1$ and $s=1=\mathcal{K}$. Then Theorem 2.1 leads to Corollaries 2.2 and 2.3 immediately.

Corollary 2.2 Under the assumption of Theorem 2.1, we have a new result for $\mathcal{K}$-fractional integral operator:

$$
\begin{aligned}
& \left(\frac{n 2^{n}}{2^{n}(n-1)+1}\right) \mathcal{P}\left(\frac{\eta_{1}+\eta_{2}}{2}\right) \\
& \quad \leq \frac{\Gamma_{\mathcal{K}}(\mathcal{K}+\alpha)}{\left(\eta_{2}-\eta_{1}\right)^{\frac{\alpha}{\mathcal{K}}}}\left[\mathcal{J}_{\eta_{1}^{+}}^{\alpha, \mathcal{K}} \mathcal{P}\left(\eta_{2}\right)+\mathcal{J}_{\eta_{2}^{-}}^{\alpha, \mathcal{K}} \mathcal{P}\left(\eta_{1}\right)\right] \\
& \quad \leq \frac{\left[\mathcal{P}\left(\eta_{1}\right)+\mathcal{P}\left(\eta_{2}\right)\right]}{n}\left[\sum_{i=1}^{n}\left[\frac{(\alpha+2 i \mathcal{K})}{(\alpha+i \mathcal{K})}-\frac{\alpha}{\mathcal{K}} \mathbb{B}\left(\frac{\alpha}{\mathcal{K}}, i+1\right)\right]\right] .
\end{aligned}
$$


Corollary 2.3 Under the assumption of Theorem 2.1, we have the following new result for $R L$-fractional integral operator:

$$
\begin{aligned}
& \left(\frac{n 2^{n}}{2^{n}(n-1)+1}\right) \mathcal{P}\left(\frac{\eta_{1}+\eta_{2}}{2}\right) \\
& \quad \leq \frac{\Gamma(\alpha+1)}{\left(\eta_{2}-\eta_{1}\right)^{\alpha}}\left[\mathcal{J}_{\eta_{1}^{+}}^{\alpha} \mathcal{P}\left(\eta_{2}\right)+\mathcal{J}_{\eta_{2}^{-}}^{\alpha} \mathcal{P}\left(\eta_{1}\right)\right] \\
& \quad \leq \frac{\left[\mathcal{P}\left(\eta_{1}\right)+\mathcal{P}\left(\eta_{2}\right)\right]}{n} \sum_{i=1}^{n}\left[\frac{\alpha+2 i}{(\alpha+i)}-\alpha \mathbb{B}(\alpha, i+1)\right] .
\end{aligned}
$$

Remark 2.4 If $s=\mathcal{K}=\alpha=1$, then Theorem 2.1 becomes Theorem 4 of [69].

\section{Ostrowski type inequalities for first-order differentiable functions}

The aim of this section is to find new estimates that refine the Ostrowski type inequality for the function whose first derivative in absolute value at a certain power is a $n$-polynomial $s$ convex function. It is remarkable that Farid and Usman [74] adopted some ideas to derive the Ostrowski type inequalities involving $\mathcal{K}$-fractional integrals.

Lemma 3.1 (See [74]) Let $\alpha \in(0,1], \mathcal{K}>0, \eta_{2}>\eta_{1}$ and $\mathcal{P}: \mathcal{I}=\left[\eta_{1}, \eta_{2}\right] \rightarrow \mathbb{R}$ be a differentiable function on $\mathcal{I}^{\circ}$ such that $\mathcal{P}^{\prime} \in L_{1}\left[\eta_{1}, \eta_{2}\right]$. Then

$$
\begin{aligned}
& \frac{\left(z-\eta_{1}\right)^{\frac{\alpha}{\mathcal{K}}}+\left(\eta_{2}-z\right)^{\frac{\alpha}{\mathcal{K}}}}{\eta_{2}-\eta_{1}} \mathcal{P}(z)-\frac{\Gamma_{\mathcal{K}}(\alpha+\mathcal{K})}{\eta_{2}-\eta_{1}}\left[\mathcal{J}_{z^{-}}^{\alpha, \mathcal{K}} \mathcal{P}\left(\eta_{1}\right)+\mathcal{J}_{z^{+}}^{\alpha, \mathcal{K}} \mathcal{P}\left(\eta_{2}\right)\right] \\
& =\frac{\left(z-\eta_{1}\right)^{\frac{\alpha}{\mathcal{K}}+1}}{\eta_{2}-\eta_{1}} \int_{0}^{1} \zeta^{\frac{\alpha}{\mathcal{K}}} \mathcal{P}^{\prime}\left(\zeta z+(1-\zeta) \eta_{1}\right) d \zeta \\
& \quad-\frac{\left(\eta_{2}-z\right)^{\frac{\alpha}{\mathcal{K}}+1}}{\eta_{2}-\eta_{1}} \int_{0}^{1} \zeta^{\frac{\alpha}{\mathcal{K}}} \mathcal{P}^{\prime}\left(\zeta z+(1-\zeta) \eta_{2}\right) d \zeta .
\end{aligned}
$$

Using Lemma 3.1, we can prove Theorem 3.2.

Theorem 3.2 Let $\alpha, \mathcal{K}>0, s \in[0,1], n \in \mathbb{N}, \eta_{2}>\eta_{1}$ and $\mathcal{P}: \mathcal{I}=\left[\eta_{1}, \eta_{2}\right] \rightarrow \mathbb{R}$ be a differentiable function on $\mathcal{I}^{\circ}$ such that $\mathcal{P}^{\prime} \in L_{1}\left[\eta_{1}, \eta_{2}\right]$ and $\left|\mathcal{P}^{\prime}(z)\right|$ is a n-polynomial s-type convex function on $\mathcal{I}$ with $\left|\mathcal{P}^{\prime}(z)\right| \leq \mathcal{M}$ for all $z \in\left[\eta_{1}, \eta_{2}\right]$. Then we have

$$
\begin{aligned}
& \left|\frac{\left(z-\eta_{1}\right)^{\frac{\alpha}{\mathcal{K}}}+\left(\eta_{2}-z\right)^{\frac{\alpha}{\mathcal{K}}}}{\eta_{2}-\eta_{1}} \mathcal{P}(z)-\frac{\Gamma_{\mathcal{K}}(\alpha+\mathcal{K})}{\eta_{2}-\eta_{1}}\left[\mathcal{J}_{z^{-}}^{\alpha, \mathcal{K}} \mathcal{P}\left(\eta_{1}\right)+\mathcal{J}_{z^{+}}^{\alpha, \mathcal{K}} \mathcal{P}\left(\eta_{2}\right)\right]\right| \\
& \leq \mathcal{M}\left[\frac{\left(z-\eta_{1}\right)^{\frac{\alpha}{\mathcal{K}}+1}+\left(\eta_{2}-z\right)^{\frac{\alpha}{\mathcal{K}}+1}}{\eta_{2}-\eta_{1}}\right] \\
& \quad \times \frac{1}{n} \sum_{i=1}^{n}\left[\left[\frac{\alpha(1-s i)+\mathcal{K}(i+1-s i)}{(\alpha+\mathcal{K})(\alpha+(i+1) \mathcal{K})}\right]+\left[\frac{\mathcal{K}}{\alpha+\mathcal{K}}-s^{i} \mathbb{B}\left(\frac{\alpha}{\mathcal{K}}+1, i+1\right)\right]\right] .
\end{aligned}
$$


Proof From Lemma 3.1 and the fact that $\left|\mathcal{P}^{\prime}\right|$ is a $n$-polynomial $s$-type convex function on $\mathcal{I}$ we clearly see that

$$
\begin{aligned}
& \left|\frac{\left(z-\eta_{1}\right)^{\frac{\alpha}{\mathcal{K}}}+\left(\eta_{2}-z\right)^{\frac{\alpha}{\mathcal{K}}}}{\eta_{2}-\eta_{1}} \mathcal{P}(z)-\frac{\Gamma_{\mathcal{K}}(\alpha+\mathcal{K})}{\eta_{2}-\eta_{1}}\left[\mathcal{J}_{z^{-}}^{\alpha, \mathcal{K}} \mathcal{P}\left(\eta_{1}\right)+\mathcal{J}_{z^{+}}^{\alpha, \mathcal{K}} \mathcal{P}\left(\eta_{2}\right)\right]\right| \\
& \leq \frac{\left(z-\eta_{1}\right)^{\frac{\alpha}{\mathcal{K}}+1}}{\eta_{2}-\eta_{1}} \int_{0}^{1} \zeta^{\frac{\alpha}{\mathcal{K}}}\left|\mathcal{P}^{\prime}\left(\zeta z+(1-\zeta) \eta_{1}\right)\right| d \zeta \\
& +\frac{\left(\eta_{2}-z\right)^{\frac{\alpha}{\mathcal{K}}+1}}{\eta_{2}-\eta_{1}} \int_{0}^{1} \zeta^{\frac{\alpha}{\mathcal{K}}}\left|\mathcal{P}^{\prime}\left(\zeta z+(1-\zeta) \eta_{2}\right)\right| d \zeta \\
& \left.\leq \frac{\left(z-\eta_{1}\right)^{\frac{\alpha}{\mathcal{K}}+1}}{\eta_{2}-\eta_{1}} \int_{0}^{1} \zeta^{\frac{\alpha}{\mathcal{K}}}\left[\frac{1}{n} \sum_{i=1}^{n}\left[1-(s \zeta)^{i}\right]\left|\mathcal{P}^{\prime}\left(\eta_{1}\right)\right|+\frac{1}{n} \sum_{i=1}^{n}\left[1-(s(1-\zeta))^{i}\right] \mid \mathcal{P}^{\prime}(z)\right]\right] d \zeta \\
& +\frac{\left(\eta_{2}-z\right)^{\frac{\alpha}{\mathcal{K}}+1}}{\eta_{2}-\eta_{1}} \int_{0}^{1} \zeta^{\frac{\alpha}{\mathcal{K}}}\left[\frac{1}{n} \sum_{i=1}^{n}\left[1-(s \zeta)^{i}\right]\left|\mathcal{P}^{\prime}\left(\eta_{2}\right)\right|\right. \\
& \left.+\frac{1}{n} \sum_{i=1}^{n}\left[1-(s(1-\zeta))^{i}\right]\left|\mathcal{P}^{\prime}(z)\right|\right] d \zeta \\
& \leq\left[\frac{\left(z-\eta_{1}\right)^{\frac{\alpha}{\mathcal{K}}+1}+\left(\eta_{2}-z\right)^{\frac{\alpha}{\mathcal{K}}+1}}{\eta_{2}-\eta_{1}}\right] \\
& \times \frac{\mathcal{M}}{n} \sum_{i=1}^{n}\left[\int_{0}^{1} \zeta^{\frac{\alpha}{\mathcal{K}}}\left[1-(s \zeta)^{i}\right] d \zeta+\int_{0}^{1} \zeta^{\frac{\alpha}{\mathcal{K}}}\left[1-(s(1-\zeta))^{i}\right] d \zeta\right] \\
& \leq\left[\frac{\left(z-\eta_{1}\right)^{\frac{\alpha}{K}+1}+\left(\eta_{2}-z\right)^{\frac{\alpha}{K}+1}}{\eta_{2}-\eta_{1}}\right] \\
& \times \frac{\mathcal{M}}{n} \sum_{i=1}^{n}\left[\left[\frac{\alpha(1-s i)+\mathcal{K}(i+1-s i)}{(\alpha+\mathcal{K})((\alpha+(i+1) \mathcal{K}))}\right]+\left[\frac{\mathcal{K}}{\alpha+\mathcal{K}}-s^{i} \mathbb{B}\left(\frac{\alpha}{\mathcal{K}+1} \cdot i+1\right)\right]\right],
\end{aligned}
$$

where we have used the facts that

$$
\int_{0}^{1} \zeta^{\frac{\alpha}{\mathcal{K}}}\left[1-(s \zeta)^{i}\right] d \zeta=\frac{\alpha(1-s i)+\mathcal{K}(i+1-s i)}{(\alpha+\mathcal{K})((\alpha+(i+1) \mathcal{K}))}
$$

and

$$
\int_{0}^{1} \zeta^{\frac{\alpha}{\mathcal{K}}}\left[1-(s(1-\zeta))^{i}\right] d \zeta=\frac{\mathcal{K}}{\alpha+\mathcal{K}}-s^{i} \mathbb{B}\left(\frac{\alpha}{\mathcal{K}}+1, i+1\right)
$$

This completes the proof.

Let $\mathcal{K}=1$ and $\mathcal{K}=\alpha=1$. Then Theorem 3.2 leads to Corollaries 3.3 and 3.4.

Corollary 3.3 Let $\alpha>0, s \in[0,1], n \in \mathbb{N}, \eta_{2}>\eta_{1}$ and $\mathcal{P}: \mathcal{I}=\left[\eta_{1}, \eta_{2}\right] \rightarrow \mathbb{R}$ be a differentiable function on $\mathcal{I}^{\circ}$ such that $\mathcal{P}^{\prime} \in L_{1}\left[\eta_{1}, \eta_{2}\right]$ and $\left|\mathcal{P}^{\prime}(z)\right|$ is a n-polynomial s-type convex 
function on $\mathcal{I}$ with $\left|\mathcal{P}^{\prime}(z)\right| \leq \mathcal{M}$ for all $z \in\left[\eta_{1}, \eta_{2}\right]$. Then one has

$$
\begin{aligned}
& \left|\frac{\left(z-\eta_{1}\right)^{\alpha}+\left(\eta_{2}-z\right)^{\alpha}}{\eta_{2}-\eta_{1}} \mathcal{P}(z)-\frac{\Gamma(\alpha+1)}{\eta_{2}-\eta_{1}}\left[\mathcal{J}_{z^{-}}^{\alpha} \mathcal{P}\left(\eta_{1}\right)+\mathcal{J}_{z^{+}}^{\alpha} \mathcal{P}\left(\eta_{2}\right)\right]\right| \\
& \leq \mathcal{M}\left[\frac{\left(z-\eta_{1}\right)^{\alpha+1}+\left(\eta_{2}-z\right)^{\alpha+1}}{\eta_{2}-\eta_{1}}\right] \\
& \quad \times \frac{1}{n} \sum_{i=1}^{n}\left\{\left[\frac{\alpha(1-s i)+(i+1-s i)}{(\alpha+1)(\alpha+(i+1))}\right]+\left[\frac{1}{\alpha+1}-s^{i} \mathbb{B}(\alpha+1, i+1)\right]\right\} .
\end{aligned}
$$

Corollary 3.4 Let $s \in[0,1], n \in \mathbb{N}, \eta_{2}>\eta_{1}$ and $\mathcal{P}: \mathcal{I}=\left[\eta_{1}, \eta_{2}\right] \rightarrow \mathbb{R}$ be a differentiable function on $\mathcal{I}^{\circ}$ such that $\mathcal{P}^{\prime} \in L_{1}\left[\eta_{1}, \eta_{2}\right]$ and $\left|\mathcal{P}^{\prime}(z)\right|$ is a n-polynomial s-type convex function on $\mathcal{I}$ with $\left|\mathcal{P}^{\prime}(z)\right| \leq \mathcal{M}$ for all $z \in\left[\eta_{1}, \eta_{2}\right]$. Then

$$
\begin{aligned}
& \left|\mathcal{P}(z)-\frac{1}{\eta_{2}-\eta_{1}} \int_{\eta_{1}}^{\eta_{2}} \mathcal{P}(z) d z\right| \\
& \quad \leq \frac{\mathcal{M}}{n}\left(\frac{\left(z-\eta_{1}\right)^{2}+\left(\eta_{2}-z\right)^{2}}{\eta_{2}-\eta_{1}}\right) \sum_{i=1}^{n}\left(\frac{4+9 i+3 i^{2}-4 s i^{2}-6 s i}{2(i+1)(i+2)}\right) .
\end{aligned}
$$

Remark 3.5 Let $\mathcal{K}=\alpha=1$ and $n=s=1$. Then Corollary 3.4 leads to inequality (1.2).

Theorem 3.6 Let $\alpha, \mathcal{K}>0, s \in[0,1], n \in \mathbb{N}, q>1, \eta_{2}>\eta_{1}, \mathcal{I}=\left[\eta_{1}, \eta_{2}\right]$, and $\mathcal{P}: \mathcal{I} \rightarrow \mathbb{R}$ be a differentiable function on $\mathcal{I}^{\circ}$ such that $\mathcal{P}^{\prime} \in L_{1}\left[\eta_{1}, \eta_{2}\right],\left|\mathcal{P}^{\prime}(z)\right|^{q}$ is a n-polynomial s-type convex function on $\mathcal{I}$ and $\left|\mathcal{P}^{\prime}(z)\right| \leq \mathcal{M}$ for all $z \in\left[\eta_{1}, \eta_{2}\right]$. Then we have

$$
\begin{aligned}
& \left|\frac{\left(z-\eta_{1}\right)^{\frac{\alpha}{\mathcal{K}}}+\left(\eta_{2}-z\right)^{\frac{\alpha}{\mathcal{K}}}}{\eta_{2}-\eta_{1}} \mathcal{P}(z)-\frac{\Gamma_{\mathcal{K}}(\alpha+\mathcal{K})}{\eta_{2}-\eta_{1}}\left[\mathcal{J}_{z^{-}}^{\alpha, \mathcal{K}} \mathcal{P}\left(\eta_{1}\right)+\mathcal{J}_{z^{+}}^{\alpha, \mathcal{K}} \mathcal{P}\left(\eta_{2}\right)\right]\right| \\
& \leq\left(\frac{\mathcal{K}}{\alpha+\mathcal{K}}\right)^{1-\frac{1}{q}}\left[\frac{\left(z-\eta_{1}\right)^{\frac{\alpha}{\mathcal{K}}+1}+\left(\eta_{2}-z\right)^{\frac{\alpha}{\mathcal{K}}+1}}{\eta_{2}-\eta_{1}}\right] \\
& \quad \times\left[\frac { \mathcal { M } ^ { q } } { n } \sum _ { i = 1 } ^ { n } \left(\left[\frac{\alpha(1-s i)+\mathcal{K}(i+1-s i)}{(\alpha+\mathcal{K})((\alpha+(i+1) \mathcal{K}))}\right]\right.\right. \\
& \left.\left.+\left[\frac{\mathcal{K}}{\alpha+\mathcal{K}}-s^{i} \mathbb{B}\left(\frac{\alpha}{\mathcal{K}}+1, i+1\right)\right]\right)\right]^{\frac{1}{q}} .
\end{aligned}
$$

Proof It follows from Lemma 3.1 and $\left|\mathcal{P}^{\prime}\right|^{q}$ is a $n$-polynomial $s$-type convex function together with the power mean inequality that

$$
\begin{aligned}
& \left|\frac{\left(z-\eta_{1}\right)^{\frac{\alpha}{\mathcal{K}}}+\left(\eta_{2}-z\right)^{\frac{\alpha}{\mathcal{K}}}}{\eta_{2}-\eta_{1}} \mathcal{P}(z)-\frac{\Gamma_{\mathcal{K}}(\alpha+\mathcal{K})}{\eta_{2}-\eta_{1}}\left[\mathcal{J}_{z^{-}}^{\alpha, \mathcal{K}} \mathcal{P}\left(\eta_{1}\right)+\mathcal{J}_{z^{+}}^{\alpha, \mathcal{K}} \mathcal{P}\left(\eta_{2}\right)\right]\right| \\
& \leq \frac{\left(z-\eta_{1}\right)^{\frac{\alpha}{\mathcal{K}}+1}}{\eta_{2}-\eta_{1}} \int_{0}^{1} \zeta^{\frac{\alpha}{\mathcal{K}}}\left|\mathcal{P}^{\prime}\left(\zeta z+(1-\zeta) \eta_{1}\right)\right| d \zeta \\
& \quad+\frac{\left(\eta_{2}-z\right)^{\frac{\alpha}{\mathcal{K}}+1}}{\eta_{2}-\eta_{1}} \int_{0}^{1} \zeta^{\frac{\alpha}{\mathcal{K}}}\left|\mathcal{P}^{\prime}\left(\zeta z+(1-\zeta) \eta_{2}\right)\right| d \zeta \\
& \leq \frac{\left(z-\eta_{1}\right)^{\frac{\alpha}{\mathcal{K}}+1}}{\eta_{2}-\eta_{1}}\left(\int_{0}^{1} \zeta^{\frac{\alpha}{\mathcal{K}}} d \zeta\right)^{1-\frac{1}{q}}\left(\int_{0}^{1} \zeta^{\frac{\alpha}{\mathcal{K}}}\left|\mathcal{P}^{\prime}\left(\zeta z+(1-\zeta) \eta_{1}\right)\right|^{q} d \zeta\right)^{\frac{1}{q}}
\end{aligned}
$$




$$
\begin{aligned}
& +\frac{\left(\eta_{2}-z\right)^{\frac{\alpha}{\mathcal{K}}+1}}{\eta_{2}-\eta_{1}}\left(\int_{0}^{1} \zeta^{\frac{\alpha}{\mathcal{K}}} d \zeta\right)^{1-\frac{1}{q}}\left(\int_{0}^{1} \zeta^{\frac{\alpha}{\mathcal{K}}}\left|\mathcal{P}^{\prime}\left(\zeta z+(1-\zeta) \eta_{2}\right)\right|^{q} d \zeta\right)^{\frac{1}{q}} \\
\leq & \left(\frac{\mathcal{K}}{\alpha+\mathcal{K}}\right)^{1-\frac{1}{q}}\left[\frac { ( z - \eta _ { 1 } ) ^ { \frac { \alpha } { \mathcal { K } } + 1 } } { \eta _ { 2 } - \eta _ { 1 } } \left(\frac{1}{n} \sum_{i=1}^{n} \int_{0}^{1} \zeta^{\frac{\alpha}{\mathcal{K}}}\left[1-(s \zeta)^{i}\right] d \zeta\left|\mathcal{P}^{\prime}\left(\eta_{1}\right)\right|^{q}\right.\right. \\
& \left.+\frac{1}{n} \sum_{i=1}^{n} \int_{0}^{1} \zeta^{\frac{\alpha}{\mathcal{K}}}\left[1-(s(1-\zeta))^{i}\right] d \zeta\left|\mathcal{P}^{\prime}(z)\right|^{q}\right)^{\frac{1}{q}} \\
& +\frac{\left(\eta_{2}-z\right)^{\frac{\alpha}{\mathcal{K}}+1}}{\eta_{2}-\eta_{1}}\left(\frac{1}{n} \sum_{i=1}^{n} \int_{0}^{1} \zeta^{\frac{\alpha}{\mathcal{K}}}\left[1-(s \zeta)^{i}\right] d \zeta\left|\mathcal{P}^{\prime}\left(\eta_{2}\right)\right|^{q}\right. \\
& \left.\left.+\frac{1}{n} \sum_{i=1}^{n} \int_{0}^{1} \zeta^{\frac{\alpha}{\mathcal{K}}}\left[1-(s(1-\zeta))^{i}\right] d \zeta\left|\mathcal{P}^{\prime}(z)\right|^{q}\right)^{\frac{1}{q}}\right] \\
\leq & \left(\frac{\mathcal{K}}{\alpha+\mathcal{K}}\right)^{1-\frac{1}{q}}\left[\frac{\left(z-\eta_{1}\right)^{\frac{\alpha}{\mathcal{K}}+1}+\left(\eta_{2}-z\right)^{\frac{\alpha}{\mathcal{K}}+1}}{\eta_{2}-\eta_{1}}\right] \\
& \times\left[\frac{\mathcal{M}^{q}}{n} \sum_{i=1}^{n}\left(\left[\frac{\alpha(1-s i)+\mathcal{K}(i+1-s i)}{(\alpha+\mathcal{K})((\alpha+(i+1) \mathcal{K}))}\right]+\left[\frac{\mathcal{K}}{\alpha+\mathcal{K}}-s^{i} \mathbb{B}\left(\frac{\alpha}{\mathcal{K}}+1, i+1\right)\right]\right)\right]^{\frac{1}{q}} .
\end{aligned}
$$

Remark 3.7 If we choose $q=1$, then Theorem 3.6 reduces to Theorem 3.2.

Theorem 3.8 Let $\alpha, \mathcal{K}>0, s \in[0,1], n \in \mathbb{N}, r, q>1$ with $1 / r+1 / q=1, \eta_{2}>\eta_{1}, \mathcal{I}=\left[\eta_{1}, \eta_{2}\right]$, and $\mathcal{P}: \mathcal{I} \rightarrow \mathbb{R}$ be a differentiable function on $\mathcal{I}^{\circ}$ such that $\mathcal{P}^{\prime} \in L_{1}\left[\eta_{1}, \eta_{2}\right],\left|\mathcal{P}^{\prime}(z)\right|^{q}$ is a n-polynomial s-type convex function on $\mathcal{I}$ and $\left|\mathcal{P}^{\prime}(z)\right| \leq \mathcal{M}$ for all $z \in\left[\eta_{1}, \eta_{2}\right]$. Then one has

$$
\begin{aligned}
& \left|\frac{\left(z-\eta_{1}\right)^{\frac{\alpha}{\mathcal{K}}}+\left(\eta_{2}-z\right)^{\frac{\alpha}{\mathcal{K}}}}{\eta_{2}-\eta_{1}} \mathcal{P}(z)-\frac{\Gamma_{\mathcal{K}}(\alpha+\mathcal{K})}{\eta_{2}-\eta_{1}}\left[\mathcal{J}_{z^{-}}^{\alpha, \mathcal{K}} \mathcal{P}\left(\eta_{1}\right)+\mathcal{J}_{z^{+}}^{\alpha, \mathcal{K}} \mathcal{P}\left(\eta_{2}\right)\right]\right| \\
& \quad \leq\left(\frac{\mathcal{K}}{r \alpha+\mathcal{K}}\right)^{\frac{1}{r}}\left[\frac{\left(z-\eta_{1}\right)^{\frac{\alpha}{\mathcal{K}}+1}+\left(\eta_{2}-z\right)^{\frac{\alpha}{\mathcal{K}}+1}}{\eta_{2}-\eta_{1}}\right] \frac{1}{n} \sum_{i=1}^{n}\left(\frac{2 \mathcal{M}^{q}\left(i+1-s^{i}\right)}{i+1}\right)^{\frac{1}{q}} .
\end{aligned}
$$

Proof Making use of Lemma 3.1 and $\left|\mathcal{P}^{\prime}\right|^{q}$ is a $n$-polynomial $s$-type convex function on $\mathcal{I}$ together with the Hölder inequality we get

$$
\begin{aligned}
& \left|\frac{\left(z-\eta_{1}\right)^{\frac{\alpha}{\mathcal{K}}}+\left(\eta_{2}-z\right)^{\frac{\alpha}{\mathcal{K}}}}{\eta_{2}-\eta_{1}} \mathcal{P}(z)-\frac{\Gamma_{\mathcal{K}}(\alpha+\mathcal{K})}{\eta_{2}-\eta_{1}}\left[\mathcal{J}_{z^{z}}^{\alpha, \mathcal{K}} \mathcal{P}\left(\eta_{1}\right)+\mathcal{J}_{z^{+}}^{\alpha, \mathcal{K}} \mathcal{P}\left(\eta_{2}\right)\right]\right| \\
& \leq \frac{\left(z-\eta_{1}\right)^{\frac{\alpha}{\mathcal{K}}+1}}{\eta_{2}-\eta_{1}} \int_{0}^{1} \zeta^{\frac{\alpha}{\mathcal{K}}}\left|\mathcal{P}^{\prime}\left(\zeta z+(1-\zeta) \eta_{1}\right)\right| d \zeta \\
& \quad+\frac{\left(\eta_{2}-z\right)^{\frac{\alpha}{\mathcal{K}}+1}}{\eta_{2}-\eta_{1}} \int_{0}^{1} \zeta^{\frac{\alpha}{\mathcal{K}}}\left|\mathcal{P}^{\prime}\left(\zeta z+(1-\zeta) \eta_{2}\right)\right| d \zeta \\
& \leq \frac{\left(z-\eta_{1}\right)^{\frac{\alpha}{\mathcal{K}}+1}}{\eta_{2}-\eta_{1}}\left(\int_{0}^{1} \zeta^{\frac{r \alpha}{\mathcal{K}}} d \zeta\right)^{\frac{1}{r}}\left(\int_{0}^{1}\left|\mathcal{P}^{\prime}\left(\zeta z+(1-\zeta) \eta_{1}\right)\right|^{q} d \zeta\right)^{\frac{1}{q}} \\
& \quad+\frac{\left(\eta_{2}-z\right)^{\frac{\alpha}{\mathcal{K}}+1}}{\eta_{2}-\eta_{1}}\left(\int_{0}^{1} \zeta^{\frac{r \alpha}{\mathcal{K}}} d \zeta\right)^{\frac{1}{r}}\left(\int_{0}^{1}\left|\mathcal{P}^{\prime}\left(\zeta z+(1-\zeta) \eta_{2}\right)\right|^{q} d \zeta\right)^{\frac{1}{q}}
\end{aligned}
$$




$$
\begin{aligned}
\leq & \left(\frac{\mathcal{K}}{r \alpha+\mathcal{K}}\right)^{\frac{1}{r}}\left[\frac { ( z - \eta _ { 1 } ) ^ { \frac { \alpha } { \mathcal { K } } + 1 } } { \eta _ { 2 } - \eta _ { 1 } } \left(\frac{1}{n} \sum_{i=1}^{n} \int_{0}^{1}\left[1-(s \zeta)^{i}\right] d \zeta\left|\mathcal{P}^{\prime}\left(\eta_{1}\right)\right|^{q}\right.\right. \\
& \left.+\frac{1}{n} \sum_{i=1}^{n} \int_{0}^{1}\left[1-(s(1-\zeta))^{i}\right] d \zeta\left|\mathcal{P}^{\prime}(z)\right|^{q}\right)^{\frac{1}{q}} \\
& +\frac{\left(\eta_{2}-z\right)^{\frac{\alpha}{\mathcal{K}}+1}}{\eta_{2}-\eta_{1}}\left(\frac{1}{n} \sum_{i=1}^{n} \int_{0}^{1}\left[1-(s \zeta)^{i}\right] d \zeta\left|\mathcal{P}^{\prime}\left(\eta_{2}\right)\right|^{q}\right. \\
& \left.\left.+\frac{1}{n} \sum_{i=1}^{n} \int_{0}^{1}\left[1-(s(1-\zeta))^{i}\right] d \zeta\left|\mathcal{P}^{\prime}(z)\right|^{q}\right)^{\frac{1}{q}}\right] \\
\leq & \left(\frac{\mathcal{K}}{r \alpha+\mathcal{K}}\right)^{\frac{1}{r}}\left[\frac{\left(z-\eta_{1}\right)^{\frac{\alpha}{\mathcal{K}}+1}+\left(\eta_{2}-z\right)^{\frac{\alpha}{K}+1}}{\eta_{2}-\eta_{1}}\right] \frac{1}{n} \sum_{i=1}^{n}\left(\frac{2 \mathcal{M}^{q}\left(i+1-s^{i}\right)}{i+1}\right)^{\frac{1}{q}}
\end{aligned}
$$

\section{New Ostrowski type inequalities for twice differentiable functions}

We first establish a fractional integral identity which is the extension of the result established by Park [75].

Lemma 4.1 Let $\alpha, \mathcal{K}>0, \eta_{2}>\eta_{1}, \mathcal{I}=\left[\eta_{1}, \eta_{2}\right]$, and $\mathcal{P}: \mathcal{I} \rightarrow \mathbb{R}$ be a twice differentiable function on $\mathcal{I}^{\circ}$ such that $\mathcal{P}^{\prime \prime} \in L_{1}\left[\eta_{1}, \eta_{2}\right]$. Then the identity

$$
\begin{aligned}
(1-\lambda) & {\left[\frac{\left(\eta_{2}-z\right)^{\frac{\alpha}{\mathcal{K}}}-\left(z-\eta_{1}\right)^{\frac{\alpha}{\mathcal{K}}}}{\eta_{2}-\eta_{1}}\right] \mathcal{P}^{\prime}(z)+\left(1+\frac{\alpha}{\mathcal{K}}-\lambda\right)\left[\frac{\left(z-\eta_{1}\right)^{\frac{\alpha}{\mathcal{K}}}+\left(\eta_{2}-z\right)^{\frac{\alpha}{\mathcal{K}}}}{\eta_{2}-\eta_{1}}\right] \mathcal{P}(z) } \\
& +\lambda\left[\frac{\left(z-\eta_{1} \frac{\alpha}{\mathcal{K}} \mathcal{P}\left(\eta_{1}\right)+\left(\eta_{2}-z\right)^{\frac{\alpha}{\mathcal{K}}} \mathcal{P}\left(\eta_{2}\right)\right.}{\eta_{2}-\eta_{1}}\right] \\
& -\frac{\Gamma_{\mathcal{K}}(\alpha+2 \mathcal{K})}{\eta_{2}-\eta_{1}}\left[\mathcal{J}_{z^{-}}^{\alpha, \mathcal{K}} \mathcal{P}\left(\eta_{1}\right)+\mathcal{J}_{z^{+}}^{\alpha, \mathcal{K}} \mathcal{P}\left(\eta_{2}\right)\right] \\
= & \frac{\left(z-\eta_{1}\right)^{\frac{\alpha}{\mathcal{K}}+2}}{\eta_{2}-\eta_{1}} \int_{0}^{1} \zeta\left(\lambda-\zeta^{\frac{\alpha}{\mathcal{K}}}\right) \mathcal{P}^{\prime \prime}\left(\zeta z+(1-\zeta) \eta_{1}\right) d \zeta \\
& +\frac{\left(\eta_{2}-\zeta\right)^{\frac{\alpha}{\mathcal{K}}+2}}{\eta_{2}-\eta_{1}} \int_{0}^{1} \zeta\left(\lambda-\zeta^{\frac{\alpha}{\mathcal{K}}}\right) \mathcal{P}^{\prime \prime}\left(\zeta z+(1-\zeta) \eta_{2}\right) d \zeta
\end{aligned}
$$

holds for $z \in\left[\eta_{1}, \eta_{2}\right]$ and $\lambda \in[0,1]$.

Proof Let $v=\zeta z+(1-\zeta) \eta_{1}$ and $v=\zeta z+(1-\zeta) \eta_{2}$, respectively. Then (1.5) and (1.6) lead to

$$
\int_{0}^{1} \zeta^{\frac{\alpha}{\mathcal{K}}-1} \mathcal{P}\left(\zeta z+(1-\zeta) \eta_{1}\right) d \zeta=\frac{\mathcal{K} \Gamma_{\mathcal{K}}(\alpha)}{\left(z-\eta_{1}\right)^{\frac{\alpha}{K}}} \mathcal{J}_{z^{-}}^{\alpha, \mathcal{K}} \mathcal{P}\left(\eta_{1}\right)
$$

and

$$
\int_{0}^{1} \zeta^{\frac{\alpha}{\mathcal{K}}-1} \mathcal{P}\left(\zeta z+(1-\zeta) \eta_{2}\right) d \zeta=\frac{\mathcal{K} \Gamma_{\mathcal{K}}(\alpha)}{\left(z-\eta_{2}\right)^{\frac{\alpha}{\mathcal{K}}}} \mathcal{J}_{z^{+}}^{\alpha, \mathcal{K}} \mathcal{P}\left(\eta_{2}\right)
$$


Integrating by parts, using (4.2) and (4.3), and changing the variables, for $z \neq \eta_{1}$ we can write

$$
\begin{aligned}
& \int_{0}^{1} \zeta\left(\lambda-\zeta^{\frac{\alpha}{\mathcal{K}}}\right) \mathcal{P}^{\prime \prime}\left(\zeta z+(1-\zeta) \eta_{1}\right) d \zeta \\
& =(\lambda-1) \frac{\mathcal{P}^{\prime}(z)}{z-\eta_{1}}+\left(1+\frac{\alpha}{\mathcal{K}}-\lambda\right) \frac{\mathcal{P}(z)}{\left(z-\eta_{1}\right)^{2}} \\
& \quad+\lambda \frac{\mathcal{P}\left(\eta_{1}\right)}{\left(z-\eta_{1}\right)^{2}}-\frac{\Gamma_{\mathcal{K}}(\alpha+2 \mathcal{K})}{\left(z-\eta_{1}\right) \frac{\alpha}{\mathcal{K}}+2} \mathcal{J}_{z^{-}}^{\alpha, \mathcal{K}} \mathcal{P}\left(\eta_{1}\right) .
\end{aligned}
$$

Similarly, for $z \neq \eta_{2}$ we get

$$
\begin{aligned}
\int_{0}^{1} \zeta\left(\lambda-\zeta^{\frac{\alpha}{\mathcal{K}}}\right) \mathcal{P}^{\prime \prime}\left(\zeta z+(1-\zeta) \eta_{2}\right) d \zeta \\
=(1-\lambda) \frac{\mathcal{P}^{\prime}(z)}{\eta_{2}-z}+\left(1+\frac{\alpha}{\mathcal{K}}-\lambda\right) \frac{\mathcal{P}(z)}{\left(\eta_{2}-z\right)^{2}} \\
\quad+\lambda \frac{\mathcal{P}\left(\eta_{2}\right)}{\left(\eta_{2}-z\right)^{2}}-\frac{\Gamma_{\mathcal{K}}(\alpha+2 \mathcal{K})}{\left.\left(\eta_{2}-z\right)\right)^{\alpha}+2} \mathcal{J}_{z^{+}}^{\alpha, \mathcal{K}} \mathcal{P}\left(\eta_{2}\right)
\end{aligned}
$$

Multiplying both sides of (4.4) and (4.5) by $\frac{\left(z-\eta_{1}\right) \frac{\alpha}{K^{+2}}}{\eta_{2}-\eta_{1}}$ and $\frac{\left(\eta_{2}-z\right) \frac{\alpha}{K^{+}}}{\eta_{2}-\eta_{1}}$, respectively, then adding the obtained identities, we obtain the desired identity (4.1).

In order to simplicity the notation, in what follows we denote

$$
\begin{aligned}
\mid \Omega_{\mathcal{P}} & \left(\lambda, \alpha, \mathcal{K} ; \eta_{1}, \eta_{2}, z\right) \mid \\
= & (1-\lambda)\left[\frac{\left(\eta_{2}-z\right)^{\frac{\alpha}{\mathcal{K}}}-\left(z-\eta_{1}\right)^{\frac{\alpha}{\mathcal{K}}}}{\eta_{2}-\eta_{1}}\right] \mathcal{P}^{\prime}(z) \\
& +\left(1+\frac{\alpha}{\mathcal{K}}-\lambda\right)\left[\frac{\left(z-\eta_{1}\right)^{\frac{\alpha}{\mathcal{K}}}+\left(\eta_{2}-z\right)^{\frac{\alpha}{\mathcal{K}}}}{\eta_{2}-\eta_{1}}\right] \mathcal{P}(z) \\
& +\lambda\left[\frac{\left(z-\eta_{1}\right)^{\frac{\alpha}{\mathcal{K}}} \mathcal{P}\left(\eta_{1}\right)+\left(\eta_{2}-z\right)^{\frac{\alpha}{\mathcal{K}}} \mathcal{P}\left(\eta_{2}\right)}{\eta_{2}-\eta_{1}}\right] \\
& -\frac{\Gamma_{\mathcal{K}}(\alpha+2 \mathcal{K})}{\eta_{2}-\eta_{1}}\left[\mathcal{J}_{z^{-}}^{\alpha, \mathcal{K}} \mathcal{P}\left(\eta_{1}\right)+\mathcal{J}_{z^{+}}^{\alpha, \mathcal{K}} \mathcal{P}\left(\eta_{2}\right)\right] .
\end{aligned}
$$

Theorem 4.2 Let $\alpha, \mathcal{K}>0, s \in[0,1], n \in \mathbb{N}, \eta_{2}>\eta_{1}, \mathcal{I}=\left[\eta_{1}, \eta_{2}\right]$, and $\mathcal{P}: \mathcal{I} \rightarrow \mathbb{R}$ be a twice differentiable function on $\mathcal{I}^{\circ}$ such that $\mathcal{P}^{\prime \prime} \in L_{1}\left[\eta_{1}, \eta_{2}\right]$ and $\left|\mathcal{P}^{\prime \prime}(z)\right|$ is a n-polynomial s-type convex function on $\mathcal{I}$. Then the inequality

$$
\begin{aligned}
& \left|\Omega_{\mathcal{P}}\left(\lambda, \alpha, \mathcal{K} ; \eta_{1}, \eta_{2}, z\right)\right| \\
& \leq\left[\frac{1}{n} \sum_{i=1}^{n} \mathcal{A}_{1}(\alpha, \mathcal{K} ; i, s)\left[\frac{\left(z-\eta_{1}\right) \frac{\alpha}{\mathcal{K}^{+2}}}{\eta_{2}-\eta_{1}}\left|\mathcal{P}^{\prime \prime}\left(\eta_{1}\right)\right|+\frac{\left(\eta_{2}-z\right) \frac{\alpha}{\mathcal{K}}+2}{\eta_{2}-\eta_{1}}\left|\mathcal{P}^{\prime \prime}\left(\eta_{2}\right)\right|\right]\right. \\
& \left.\quad+\frac{\left(z-\eta_{1}\right) \frac{\alpha}{\mathcal{K}^{+2}}+\left(\eta_{2}-z\right) \frac{\alpha}{\mathcal{K}}+2}{n\left(\eta_{2}-\eta_{1}\right)} \sum_{i=1}^{n} \mathcal{A}_{2}(\alpha, \mathcal{K} ; i, s)\left|\mathcal{P}^{\prime \prime}(z)\right|\right]
\end{aligned}
$$


holds for all $z \in\left[\eta_{1}, \eta_{2}\right]$, where

$$
\begin{aligned}
\mathcal{A}_{1}(\alpha, \mathcal{K} ; i, s) & =\int_{0}^{1} \zeta\left(\lambda-\zeta^{\frac{\alpha}{\mathcal{K}}}\right)\left[\left(1-(s \zeta)^{i}\right)\right] d \zeta \\
& =\left[\frac{\lambda \alpha-2 \mathcal{K}(1-\lambda)}{2(\alpha+2 \mathcal{K})}-s^{i} \frac{\lambda \alpha-\mathcal{K}(i+2)(1-\lambda)}{(i+2)(\alpha+\mathcal{K}(i+2))}\right]
\end{aligned}
$$

and

$$
\begin{aligned}
\mathcal{A}_{2}(\alpha, \mathcal{K} ; i, s) & =\int_{0}^{1} \zeta\left(\lambda-\zeta^{\frac{\alpha}{\mathcal{K}}}\right)\left[1-(s(1-\zeta))^{i}\right] d \zeta \\
& =\left[\frac{\lambda \alpha-2 \mathcal{K}(1-\lambda)}{2(\alpha+2 \mathcal{K})}-s^{i}\left[\frac{\lambda}{(i+2)(i+1)}-\frac{\Gamma\left(\frac{\alpha}{\mathcal{K}}+2\right) \Gamma(i+1)}{\Gamma\left(\frac{\alpha}{\mathcal{K}}+i+3\right)}\right]\right] .
\end{aligned}
$$

Proof Making use of Lemma 4.1, the property of the modulus and the $n$-polynomial $s$-type convexity of $\left|\mathcal{P}^{\prime \prime}\right|$ on $\mathcal{I}$, we have

$$
\begin{aligned}
& \mid(1-\lambda)\left[\frac{\left(\eta_{2}-z\right)^{\frac{\alpha}{K}}-\left(z-\eta_{1}\right)^{\frac{\alpha}{K}}}{\eta_{2}-\eta_{1}}\right] \mathcal{P}^{\prime}(z)+\left(1+\frac{\alpha}{\mathcal{K}}-\lambda\right)\left[\frac{\left(z-\eta_{1}\right)^{\frac{\alpha}{K}}+\left(\eta_{2}-z\right)^{\frac{\alpha}{K}}}{\eta_{2}-\eta_{1}}\right] \mathcal{P}(z) \\
& +\lambda\left[\frac{\left(z-\eta_{1}\right)^{\frac{\alpha}{\mathcal{K}}} \mathcal{P}\left(\eta_{1}\right)+\left(\eta_{2}-z\right)^{\frac{\alpha}{\mathcal{K}}} \mathcal{P}\left(\eta_{2}\right)}{\eta_{2}-\eta_{1}}\right] \\
& -\frac{\Gamma_{\mathcal{K}}(\alpha+2 \mathcal{K})}{\eta_{2}-\eta_{1}}\left[\mathcal{J}_{z^{+}}^{\alpha, \mathcal{K}} \mathcal{P}\left(\eta_{1}\right)+\mathcal{J}_{z^{-}}^{\alpha, \mathcal{K}} \mathcal{P}\left(\eta_{2}\right)\right] \\
& \leq \frac{\left(z-\eta_{1}\right) \frac{\alpha}{\mathcal{K}}+2}{\eta_{2}-\eta_{1}} \int_{0}^{1} \zeta\left(\lambda-\zeta^{\frac{\alpha}{\mathcal{K}}}\right)\left|\mathcal{P}^{\prime \prime}\left(\zeta z+(1-\zeta) \eta_{1}\right)\right| d \zeta \\
& +\frac{\left(\eta_{2}-\zeta\right)^{\frac{\alpha}{\mathcal{K}}+1}}{\eta_{2}-\eta_{1}} \int_{0}^{1} \zeta\left(\lambda-\zeta^{\frac{\alpha}{K}}\right)\left|\mathcal{P}^{\prime \prime}\left(\zeta z+(1-\zeta) \eta_{2}\right)\right| d \zeta \\
& \leq \frac{\left(z-\eta_{1}\right)^{\frac{\alpha}{\mathcal{K}}+2}}{\eta_{2}-\eta_{1}} \int_{0}^{1} \zeta\left(\lambda-\zeta^{\frac{\alpha}{K}}\right)\left[\frac{1}{n} \sum_{i=1}^{n}\left(1-(s \zeta)^{i}\right)\left|\mathcal{P}^{\prime \prime}\left(\eta_{1}\right)\right|\right. \\
& \left.+\frac{1}{n} \sum_{i=1}^{n}\left[1-(s(1-\zeta))^{i}\right]\left|\mathcal{P}^{\prime \prime}(z)\right|\right] d \zeta \\
& +\frac{\left(\eta_{2}-z\right)^{\frac{\alpha}{\mathcal{K}}+2}}{\eta_{2}-\eta_{1}} \int_{0}^{1} \zeta\left(\lambda-\zeta^{\frac{\alpha}{\mathcal{K}}}\right)\left[\frac{1}{n} \sum_{i=1}^{n}\left(1-(s \zeta)^{i}\right)\left|\mathcal{P}^{\prime \prime}\left(\eta_{2}\right)\right|\right. \\
& \left.+\frac{1}{n} \sum_{i=1}^{n}\left[1-(s(1-\zeta))^{i}\right]\left|\mathcal{P}^{\prime \prime}(z)\right|\right] d \zeta \\
& =\frac{\left(z-\eta_{1}\right)^{\frac{\alpha}{\mathcal{K}}+2}}{n\left(\eta_{2}-\eta_{1}\right)} \sum_{i=1}^{n}\left[\mathcal{A}_{1}(\alpha, \mathcal{K} ; i, s)\left|\mathcal{P}^{\prime \prime}\left(\eta_{1}\right)\right|+\mathcal{A}_{2}(\alpha, \mathcal{K} ; i, s)\left|\mathcal{P}^{\prime \prime}(z)\right|\right] \\
& +\frac{\left(\eta_{2}-z\right)^{\frac{\alpha}{\mathcal{K}}+2}}{n\left(\eta_{2}-\eta_{1}\right)} \sum_{i=1}^{n}\left[\mathcal{A}_{1}(\alpha, \mathcal{K} ; i, s)\left|\mathcal{P}^{\prime \prime}\left(\eta_{2}\right)\right|+\mathcal{A}_{2}(\alpha, \mathcal{K} ; i, s)\left|\mathcal{P}^{\prime \prime}(z)\right|\right] \\
& =\left[\frac{1}{n} \sum_{i=1}^{n} \mathcal{A}_{1}(\alpha, \mathcal{K} ; i, s)\left[\frac{\left(z-\eta_{1}\right)^{\frac{\alpha}{\mathcal{K}}+2}}{\eta_{2}-\eta_{1}}\left|\mathcal{P}^{\prime \prime}\left(\eta_{1}\right)\right|+\frac{\left(\eta_{2}-z\right)^{\frac{\alpha}{\mathcal{K}}+2}}{\eta_{2}-\eta_{1}}\left|\mathcal{P}^{\prime \prime}\left(\eta_{2}\right)\right|\right]\right. \\
& \left.+\frac{\left(z-\eta_{1}\right)^{\frac{\alpha}{K}+2}+\left(\eta_{2}-z\right)^{\frac{\alpha}{K}}+2}{n\left(\eta_{2}-\eta_{1}\right)} \sum_{i=1}^{n} \mathcal{A}_{2}(\alpha, \mathcal{K} ; i, s)\left|\mathcal{P}^{\prime \prime}(z)\right|\right] .
\end{aligned}
$$


Theorem 4.3 Let $\alpha, \mathcal{K}>0, s \in[0,1], q>1, n \in \mathbb{N}, \eta_{2}>\eta_{1}, \mathcal{I}=\left[\eta_{1}, \eta_{2}\right]$, and $\mathcal{P}: \mathcal{I} \rightarrow \mathbb{R}$ bea twice differentiable function on $\mathcal{I}^{\circ}$ such that $\mathcal{P}^{\prime \prime} \in L_{1}\left[\eta_{1}, \eta_{2}\right]$ and $\left|\mathcal{P}^{\prime \prime}(z)\right|^{q}$ is a n-polynomial s-type convex function on $\mathcal{I}$. Then the inequality

$$
\begin{aligned}
& \left|\Omega_{\mathcal{P}}\left(\lambda, \alpha, \mathcal{K} ; \eta_{1}, \eta_{2}, z\right)\right| \\
& \leq \mathcal{A}_{3}^{1-\frac{1}{q}}(\alpha, \mathcal{K}, \lambda)\left[\frac{\left(z-\eta_{1}\right) \mathcal{K}^{+2}}{n\left(\eta_{2}-\eta_{1}\right)} \sum_{i=1}^{n}\left(\mathcal{A}_{1}(\alpha, \mathcal{K} ; i, s)\left|\mathcal{P}^{\prime \prime}\left(\eta_{1}\right)\right|^{q}+\mathcal{A}_{2}(\alpha, \mathcal{K} ; i, s)\left|\mathcal{P}^{\prime \prime}(z)\right|^{q}\right)^{\frac{1}{q}}\right. \\
& \left.\quad+\frac{\left(\eta_{2}-z\right) \frac{\alpha}{\mathcal{K}}+2}{n\left(\eta_{2}-\eta_{1}\right)} \sum_{i=1}^{n}\left(\mathcal{A}_{1}(\alpha, \mathcal{K} ; i, s)\left|\mathcal{P}^{\prime \prime}\left(\eta_{2}\right)\right|^{q}+\mathcal{A}_{2}(\alpha, \mathcal{K} ; i, s)\left|\mathcal{P}^{\prime \prime}(z)\right|^{q}\right)^{\frac{1}{q}}\right]
\end{aligned}
$$

holds for all $z \in\left(\eta_{1}, \eta_{2}\right)$, where

$$
\begin{aligned}
& \mathcal{A}_{3}(\alpha, \mathcal{K}, \lambda) \\
& =\int_{0}^{1}\left(\zeta\left(\lambda-\zeta \frac{\alpha}{\mathcal{K}}\right)\right)^{q} d \zeta \\
& =\frac{\mathcal{K} \lambda \frac{(1+q) \mathcal{K}+\alpha q}{\alpha}}{\alpha}\left[\Gamma(1+q) \Gamma\left(\frac{\mathcal{K}(1+q)+\alpha}{\alpha}\right){ }_{2} \mathcal{F}_{1}\left(1,1+q, 2+q+\frac{\mathcal{K}(q+1)}{\alpha}, 1\right)\right. \\
& \left.\quad+\mathbb{B}\left(1+q,-\frac{\mathcal{K}(1+q)+\alpha q}{\alpha}\right)-\mathbb{B}\left(\lambda, 1+q,-\frac{(1+q) \mathcal{K}+\alpha q}{\alpha}\right)\right],
\end{aligned}
$$

and $\mathcal{A}_{1}(\alpha, \mathcal{K} ; i, s)$ and $\mathcal{A}_{2}(\alpha, \mathcal{K} ; i, s)$ are given in (4.7) and (4.8), respectively.

Proof Using Lemma 4.1, the power mean inequality and the $n$-polynomial $s$-type convexity, we have

$$
\begin{aligned}
& \mid(1-\lambda)\left[\frac{\left(\eta_{2}-z\right)^{\frac{\alpha}{\mathcal{K}}}-\left(z-\eta_{1}\right)^{\frac{\alpha}{\mathcal{K}}}}{\eta_{2}-\eta_{1}}\right] \mathcal{P}^{\prime}(z)+\left(1+\frac{\alpha}{\mathcal{K}}-\lambda\right)\left[\frac{\left(z-\eta_{1}\right)^{\frac{\alpha}{\mathcal{K}}}+\left(\eta_{2}-z\right)^{\frac{\alpha}{\mathcal{K}}}}{\eta_{2}-\eta_{1}}\right] \mathcal{P}(z) \\
&+\lambda\left[\frac{\left(z-\eta_{1}\right)^{\frac{\alpha}{\mathcal{K}}} \mathcal{P}\left(\eta_{1}\right)+\left(\eta_{2}-z\right)^{\frac{\alpha}{\mathcal{K}}} \mathcal{P}\left(\eta_{2}\right)}{\eta_{2}-\eta_{1}}\right] \\
&-\frac{\Gamma_{\mathcal{K}}(\alpha+2 \mathcal{K})}{\eta_{2}-\eta_{1}}\left[\mathcal{J}_{z^{+}}^{\alpha, \mathcal{K}} \mathcal{P}\left(\eta_{1}\right)+\mathcal{J}_{z^{-}}^{\alpha, \mathcal{K}} \mathcal{P}\left(\eta_{2}\right)\right] \mid \\
& \leq \frac{\left(z-\eta_{1}\right)^{\frac{\alpha}{\mathcal{K}}+2}}{\eta_{2}-\eta_{1}} \int_{0}^{1}\left|\zeta\left(\lambda-\zeta^{\frac{\alpha}{\mathcal{K}}}\right)\right|\left|\mathcal{P}^{\prime \prime}\left(\zeta z+(1-\zeta) \eta_{1}\right)\right| d \zeta \\
&+\frac{\left(\eta_{2}-\zeta\right)^{\frac{\alpha}{\mathcal{K}}+1}}{\eta_{2}-\eta_{1}} \int_{0}^{1}\left|\zeta\left(\lambda-\zeta^{\frac{\alpha}{\mathcal{K}}}\right)\right|\left|\mathcal{P}^{\prime \prime}\left(\zeta z+(1-\zeta) \eta_{2}\right)\right| d \zeta \\
& \leq\left(\int_{0}^{1} \zeta^{q}\left(\lambda-\zeta^{\frac{\alpha}{\mathcal{K}}}\right)^{q} d \zeta\right)^{1-\frac{1}{q}}\left[\frac{\left(z-\eta_{1}\right)^{\frac{\alpha}{\mathcal{K}}+2}}{\eta_{2}-\eta_{1}}\right. \\
& \quad \times\left(\int _ { 0 } ^ { 1 } \zeta ( \lambda - \zeta ^ { \frac { \alpha } { \mathcal { K } } } ) \left[\frac{1}{n} \sum_{i=1}^{n}\left(1-(s \zeta)^{i}\right)\left|\mathcal{P}^{\prime \prime}\left(\eta_{1}\right)\right|^{q}\right.\right. \\
&\left.\left.\quad+\frac{1}{n} \sum_{i=1}^{n}\left[1-(s(1-\zeta))^{i}\right]\left|\mathcal{P}^{\prime \prime}(z)\right|^{q}\right] d \zeta\right)^{\frac{1}{q}}
\end{aligned}
$$




$$
\begin{aligned}
& +\frac{\left(\eta_{2}-z\right)^{\frac{\alpha}{\mathcal{K}}+2}}{\eta_{2}-\eta_{1}}\left(\int _ { 0 } ^ { 1 } \zeta ( \lambda - \zeta ^ { \frac { \alpha } { \mathcal { K } } } ) \left[\frac{1}{n} \sum_{i=1}^{n}\left(1-(s \zeta)^{i}\right)\left|\mathcal{P}^{\prime \prime}\left(\eta_{2}\right)\right|^{q}\right.\right. \\
& \left.\left.\left.+\frac{1}{n} \sum_{i=1}^{n}\left[1-(s(1-\zeta))^{i}\right]\left|\mathcal{P}^{\prime \prime}(z)\right|^{q}\right] d \zeta\right)^{\frac{1}{q}}\right] \\
& =\mathcal{A}_{3}^{1-\frac{1}{q}}(\alpha, \mathcal{K}, \lambda)\left[\frac{\left(z-\eta_{1} \frac{\alpha}{\mathcal{K}}+2\right.}{n\left(\eta_{2}-\eta_{1}\right)} \sum_{i=1}^{n}\left(\mathcal{A}_{1}(\alpha, \mathcal{K} ; i, s)\left|\mathcal{P}^{\prime \prime}\left(\eta_{1}\right)\right|^{q}+\mathcal{A}_{2}(\alpha, \mathcal{K} ; i, s)\left|\mathcal{P}^{\prime \prime}(z)\right|^{q}\right)^{\frac{1}{q}}\right. \\
& \left.+\frac{\left(\eta_{2}-z\right)^{\frac{\alpha}{\mathcal{K}}+2}}{n\left(\eta_{2}-\eta_{1}\right)} \sum_{i=1}^{n}\left(\mathcal{A}_{1}(\alpha, \mathcal{K} ; i, s)\left|\mathcal{P}^{\prime \prime}\left(\eta_{2}\right)\right|^{q}+\mathcal{A}_{2}(\alpha, \mathcal{K} ; i, s)\left|\mathcal{P}^{\prime \prime}(z)\right|^{q}\right)^{\frac{1}{q}}\right]
\end{aligned}
$$

Theorem 4.4 Let $\alpha, \mathcal{K}>0, s \in[0,1], q, r>1$ with $1 / q+1 / r=1, n \in \mathbb{N}, \eta_{2}>\eta_{1}, \mathcal{I}=\left[\eta_{1}, \eta_{2}\right]$, and $\mathcal{P}: \mathcal{I} \rightarrow \mathbb{R}$ be a twice differentiable function on $\mathcal{I}^{\circ}$ such that $\mathcal{P}^{\prime \prime} \in L_{1}\left[\eta_{1}, \eta_{2}\right]$ and $\left|\mathcal{P}^{\prime \prime}(z)\right|^{q}$ is a n-polynomial s-type convex function on $\mathcal{I}$. Then the inequality

$$
\begin{aligned}
& \left|\Omega_{\mathcal{P}}\left(\lambda, \alpha, \mathcal{K} ; \eta_{1}, \eta_{2}, z\right)\right| \\
& \leq \mathcal{A}_{3}^{\frac{1}{r}}(\alpha, \mathcal{K}, \lambda)\left[\frac{\left(z-\eta_{1}\right)^{\frac{\alpha}{\mathcal{K}}+2}}{\left(\eta_{2}-\eta_{1}\right)}\left(\frac{1}{n} \sum_{i=1}^{n}\left(\frac{i+1-s^{i}}{i+1}\right)\left[\left|\mathcal{P}^{\prime \prime}(z)\right|^{q}+\left|\mathcal{P}^{\prime \prime}\left(\eta_{1}\right)\right|^{q}\right]\right)^{\frac{1}{q}}\right. \\
& \left.\quad+\frac{\left(\eta_{2}-z\right) \frac{\alpha}{\mathcal{K}}+2}{\left(\eta_{2}-\eta_{1}\right)}\left(\frac{1}{n} \sum_{i=1}^{n}\left(\frac{i+1-s^{i}}{i+1}\right)\left[\left|\mathcal{P}^{\prime \prime}(z)\right|^{q}+\left|\mathcal{P}^{\prime \prime}\left(\eta_{2}\right)\right|^{q}\right]\right)^{\frac{1}{q}}\right]
\end{aligned}
$$

holds for all $z \in\left(\eta_{1}, \eta_{2}\right)$, where $\mathcal{A}_{1}(\alpha, \mathcal{K} ; i, s), \mathcal{A}_{2}(\alpha, \mathcal{K} ; i, s)$ and $\mathcal{A}_{3}(\alpha, \mathcal{K} ; \lambda)$ are given in (4.7), (4.8) and (4.9), respectively.

Proof Using Lemma 4.1 and the Hölder inequality together with the $n$-polynomial $s$-type convexity, we have

$$
\begin{aligned}
\mid(1- & \lambda)\left[\frac{\left(\eta_{2}-z\right)^{\frac{\alpha}{\mathcal{K}}}-\left(z-\eta_{1}\right)^{\frac{\alpha}{\mathcal{K}}}}{\eta_{2}-\eta_{1}}\right] \mathcal{P}^{\prime}(z)+\left(1+\frac{\alpha}{\mathcal{K}}-\lambda\right)\left[\frac{\left(z-\eta_{1}\right)^{\frac{\alpha}{\mathcal{K}}}+\left(\eta_{2}-z\right)^{\frac{\alpha}{\mathcal{K}}}}{\eta_{2}-\eta_{1}}\right] \mathcal{P}(z) \\
& +\lambda\left[\frac{\left(z-\eta_{1}\right)^{\frac{\alpha}{\mathcal{K}}} \mathcal{P}\left(\eta_{1}\right)+\left(\eta_{2}-z\right)^{\frac{\alpha}{\mathcal{K}}} \mathcal{P}\left(\eta_{2}\right)}{\eta_{2}-\eta_{1}}\right] \\
& -\frac{\Gamma_{\mathcal{K}}(\alpha+2 \mathcal{K})}{\eta_{2}-\eta_{1}}\left[\mathcal{J}_{z^{+}}^{\alpha, \mathcal{K}} \mathcal{P}\left(\eta_{1}\right)+\mathcal{J}_{z^{-}}^{\alpha, \mathcal{K}} \mathcal{P}\left(\eta_{2}\right)\right] \mid \\
\leq & \frac{\left(z-\eta_{1}\right)^{\frac{\alpha}{\mathcal{K}}+2}}{\eta_{2}-\eta_{1}} \int_{0}^{1}\left|\zeta\left(\lambda-\zeta^{\frac{\alpha}{\mathcal{K}}}\right)\right|\left|\mathcal{P}^{\prime \prime}\left(\zeta z+(1-\zeta) \eta_{1}\right)\right| d \zeta \\
& +\frac{\left(\eta_{2}-\zeta\right)^{\frac{\alpha}{\mathcal{K}}+1}}{\eta_{2}-\eta_{1}} \int_{0}^{1}\left|\zeta\left(\lambda-\zeta^{\frac{\alpha}{\mathcal{K}}}\right)\right|\left|\mathcal{P}^{\prime \prime}\left(\zeta z+(1-\zeta) \eta_{2}\right)\right| d \zeta \\
\leq & \frac{\left(z-\eta_{1}\right)^{\frac{\alpha}{\mathcal{K}}+2}}{\eta_{2}-\eta_{1}}\left(\int_{0}^{1}\left|\zeta\left(\lambda-\zeta^{\frac{\alpha}{\mathcal{K}}}\right)\right|^{r} d \zeta\right)^{\frac{1}{r}}\left(\int_{0}^{1}\left|\mathcal{P}^{\prime \prime}\left(\zeta z+(1-\zeta) \eta_{1}\right)\right|^{q} d \zeta\right)^{\frac{1}{q}} \\
& +\frac{\left(\eta_{2}-\zeta\right)^{\frac{\alpha}{\mathcal{K}}+2}}{\eta_{2}-\eta_{1}}\left(\int_{0}^{1}\left|\zeta\left(\lambda-\zeta^{\frac{\alpha}{\mathcal{K}}}\right)\right|^{r} d \zeta\right)^{\frac{1}{r}}\left(\int_{0}^{1}\left|\mathcal{P}^{\prime \prime}\left(\zeta z+(1-\zeta) \eta_{2}\right)\right| d \zeta\right)^{\frac{1}{q}} \\
\leq & \mathcal{A}_{3}^{\frac{1}{r}}(\alpha, \mathcal{K} ; \lambda)\left[\frac { ( z - \eta _ { 1 } ) ^ { \frac { \alpha } { \mathcal { K } } + 2 } } { \eta _ { 2 } - \eta _ { 1 } } \left(\int _ { 0 } ^ { 1 } \left[\frac{1}{n} \sum_{i=1}^{n}\left(1-(s \zeta)^{i}\right)\left|\mathcal{P}^{\prime \prime}\left(\eta_{1}\right)\right|^{q}\right.\right.\right.
\end{aligned}
$$




$$
\begin{aligned}
& \left.\left.+\frac{1}{n} \sum_{i=1}^{n}\left[1-(s(1-\zeta))^{i}\right]\left|\mathcal{P}^{\prime \prime}(z)\right|^{q}\right] d \zeta\right)^{\frac{1}{q}} \\
& +\frac{\left(\eta_{2}-z\right)^{\frac{\alpha}{\mathcal{K}}+2}}{\eta_{2}-\eta_{1}}\left(\int _ { 0 } ^ { 1 } \left[\frac{1}{n} \sum_{i=1}^{n}\left(1-(s \zeta)^{i}\right)\left|\mathcal{P}^{\prime \prime}\left(\eta_{2}\right)\right|^{q}\right.\right. \\
& \left.\left.\left.+\frac{1}{n} \sum_{i=1}^{n}\left[1-(s(1-\zeta))^{i}\right]\left|\mathcal{P}^{\prime \prime}(z)\right|^{q}\right] d \zeta\right)^{\frac{1}{q}}\right] \\
& =\mathcal{A}_{3}^{\frac{1}{r}}(\alpha, \mathcal{K}, \lambda)\left[\frac{\left(z-\eta_{1} \frac{\alpha}{\mathcal{K}}+2\right.}{\left(\eta_{2}-\eta_{1}\right)}\left(\frac{1}{n} \sum_{i=1}^{n}\left(\frac{i+1-s^{i}}{i+1}\right)\left[\left|\mathcal{P}^{\prime \prime}(z)\right|^{q}+\left|\mathcal{P}^{\prime \prime}\left(\eta_{1}\right)\right|^{q}\right]\right)^{\frac{1}{q}}\right. \\
& \left.+\frac{\left(\eta_{2}-z\right)^{\frac{\alpha}{\mathcal{K}}+2}}{\left(\eta_{2}-\eta_{1}\right)}\left(\frac{1}{n} \sum_{i=1}^{n}\left(\frac{i+1-s^{i}}{i+1}\right)\left[\left|\mathcal{P}^{\prime \prime}(z)\right|^{q}+\left|\mathcal{P}^{\prime \prime}\left(\eta_{2}\right)\right|^{q}\right]\right)^{\frac{1}{q}}\right] .
\end{aligned}
$$

\section{Applications}

In this section, we provide some applications to the estimations of $\mathcal{K}$-fractional integrals, and the midpoint, trapezoidal and Simpson type inequalities for twice differentiable $n$ polynomial $s$-type convex functions by use of our results.

Let $\lambda=0$ and $z=\frac{\eta_{1}+\eta_{2}}{2}, \lambda=1$ and $z=\frac{\eta_{1}+\eta_{2}}{2}, \lambda=\frac{1}{2}$ and $z=\frac{\eta_{1}+\eta_{2}}{2}, \lambda=\frac{1}{3}$ and $z=\frac{\eta_{1}+\eta_{2}}{2}$. Then Theorem 4.2 leads to Corollaries 5.1-5.4 immediately.

Corollary 5.1 Let $\alpha, \mathcal{K}>0, s \in[0,1], n \in \mathbb{N}, \eta_{2}>\eta_{1}$, and $\mathcal{P}: \mathcal{I}=\left[\eta_{1}, \eta_{2}\right] \rightarrow \mathbb{R}$ be a twice differentiable function on $\mathcal{I}^{\circ}$ such that $\mathcal{P}^{\prime \prime} \in L_{1}\left[\eta_{1}, \eta_{2}\right]$ and $\left|\mathcal{P}^{\prime \prime}(z)\right|$ is a n-polynomial s-type convex function on $\mathcal{I}^{\circ}$. Then we have

$$
\begin{aligned}
& \left|\Omega_{\mathcal{P}}\left(0, \alpha, \mathcal{K} ; \eta_{1}, \eta_{2}, \frac{\eta_{1}+\eta_{2}}{2}\right)\right| \\
& \leq\left(\frac{\left(\eta_{2}-\eta_{1}\right)^{\frac{\alpha}{\mathcal{K}}+1}}{2^{\frac{\alpha}{\mathcal{K}}+1} n}\right)\left[\sum_{i=1}^{n} \mathcal{A}_{1}(\alpha, \mathcal{K} ; i, s)\left[\left|\mathcal{P}^{\prime \prime}\left(\eta_{1}\right)\right|+\left|\mathcal{P}^{\prime \prime}\left(\eta_{2}\right)\right|\right]\right. \\
& \left.\quad+\sum_{i=1}^{n} \mathcal{A}_{2}(\alpha, \mathcal{K} ; i, s)\left|\mathcal{P}^{\prime \prime}\left(\frac{\eta_{1}+\eta_{2}}{2}\right)\right|\right]
\end{aligned}
$$

where $\mathcal{A}_{1}(\alpha, \mathcal{K} ; i, s)$ and $\mathcal{A}_{2}(\alpha, \mathcal{K} ; i, s)$ are given in (4.7) and (4.8), respectively.

Corollary 5.2 Let $\alpha, \mathcal{K}>0, s \in[0,1], n \in \mathbb{N}, \eta_{2}>\eta_{1}$, and $\mathcal{P}: \mathcal{I}=\left[\eta_{1}, \eta_{2}\right] \rightarrow \mathbb{R}$ be a twice differentiable function on $\mathcal{I}^{\circ}$ such that $\mathcal{P}^{\prime \prime} \in L_{1}\left[\eta_{1}, \eta_{2}\right]$ and $\left|\mathcal{P}^{\prime \prime}(z)\right|$ is a n-polynomial s-type convex function on $\mathcal{I}^{\circ}$. Then one has

$$
\begin{aligned}
& \left|\Omega_{\mathcal{P}}\left(1, \alpha, \mathcal{K} ; \eta_{1}, \eta_{2}, \frac{\eta_{1}+\eta_{2}}{2}\right)\right| \\
& \quad \leq\left(\frac{\left(\eta_{2}-\eta_{1}\right)^{\frac{\alpha}{\mathcal{K}}+1}}{2^{\frac{\alpha}{\mathcal{K}}+1} n}\right)\left[\sum_{i=1}^{n}\left[\frac{\alpha}{2(\alpha+2 \mathcal{K})}-s^{i}\left(\frac{1}{(i+1)(i+2)}-\frac{\Gamma\left(\frac{\alpha}{\mathcal{K}}+2\right) \Gamma(i+1)}{\Gamma\left(\frac{\alpha}{\mathcal{K}}+i+3\right)}\right)\right]\right.
\end{aligned}
$$




$$
\begin{aligned}
& \times\left|\mathcal{P}^{\prime \prime}\left(\frac{\eta_{1}+\eta_{2}}{2}\right)\right| \\
& \left.+\sum_{i=1}^{n}\left(\frac{\alpha}{2(\alpha+2 \mathcal{K})}-\frac{s^{i} \alpha}{(i+2)(\alpha+\mathcal{K}(i+2))}\right)\left(\left|\mathcal{P}^{\prime \prime}\left(\eta_{1}\right)\right|+\left|\mathcal{P}^{\prime \prime}\left(\eta_{2}\right)\right|\right)\right]
\end{aligned}
$$

Corollary 5.3 Let $\alpha, \mathcal{K}>0, s \in[0,1], n \in \mathbb{N}, \eta_{2}>\eta_{1}$, and $\mathcal{P}: \mathcal{I}=\left[\eta_{1}, \eta_{2}\right] \rightarrow \mathbb{R}$ be a twice differentiable function on $\mathcal{I}^{\circ}$ such that $\mathcal{P}^{\prime \prime} \in L_{1}\left[\eta_{1}, \eta_{2}\right]$ and $\left|\mathcal{P}^{\prime \prime}(z)\right|$ is a n-polynomial s-type convex function on $\mathcal{I}^{\circ}$. Then

$$
\begin{aligned}
\mid \Omega_{\mathcal{P}} & \left(\frac{1}{2}, \alpha, \mathcal{K} ; \eta_{1}, \eta_{2}, \frac{\eta_{1}+\eta_{2}}{2}\right) \mid \\
\leq & \left(\frac{\left(\eta_{2}-\eta_{1}\right)^{\frac{\alpha}{\mathcal{K}}+1}}{2^{\frac{\alpha}{\mathcal{K}}+1} n}\right)\left[\sum_{i=1}^{n}\left[\frac{\alpha-2 \mathcal{K}}{4(\alpha+2 \mathcal{K})}-\frac{s^{i}[\alpha-\mathcal{K}(i+2)]}{2(i+2)(\alpha+\mathcal{K}(i+2))}\right]\left[\left|\mathcal{P}^{\prime \prime}\left(\eta_{1}\right)\right|+\left|\mathcal{P}^{\prime \prime}\left(\eta_{2}\right)\right|\right]\right. \\
& \left.+\sum_{i=1}^{n}\left[\frac{\alpha-2 \mathcal{K}}{4(\alpha+2 \mathcal{K})}-s^{i}\left(\frac{1}{2(i+1)(i+2)}-\frac{\Gamma\left(\frac{\alpha}{\mathcal{K}}+2\right) \Gamma(i+1)}{\Gamma\left(\frac{\alpha}{\mathcal{K}}+i+3\right)}\right)\right]\left|\mathcal{P}^{\prime \prime}\left(\frac{\eta_{1}+\eta_{2}}{2}\right)\right|\right] .
\end{aligned}
$$

Corollary 5.4 Let $\alpha, \mathcal{K}>0, s \in[0,1], n \in \mathbb{N}, \eta_{2}>\eta_{1}$, and $\mathcal{P}: \mathcal{I}=\left[\eta_{1}, \eta_{2}\right] \rightarrow \mathbb{R}$ be a twice differentiable function on $\mathcal{I}^{\circ}$ such that $\mathcal{P}^{\prime \prime} \in L_{1}\left[\eta_{1}, \eta_{2}\right]$ and $\left|\mathcal{P}^{\prime \prime}(z)\right|$ is a n-polynomial s-type convex function on $\mathcal{I}^{\circ}$. Then we get

$$
\begin{aligned}
& \mid \Omega_{\mathcal{P}}\left(\frac{1}{3}, \alpha, \mathcal{K} ; \eta_{1}, \eta_{2}, \frac{\eta_{1}+\eta_{2}}{2}\right) \mid \\
& \leq\left(\frac{\left(\eta_{2}-\eta_{1}\right)^{\frac{\alpha}{\mathcal{K}}+1}}{2^{\frac{\alpha}{\mathcal{K}}+1} n}\right)\left[\sum_{i=1}^{n}\left[\frac{\alpha-4 \mathcal{K}}{6(\alpha+2 \mathcal{K})}-\frac{s^{i}[\alpha-2 \mathcal{K}(i+2)]}{3(i+2)(\alpha+\mathcal{K}(i+2))}\right]\left(\left|\mathcal{P}^{\prime \prime}\left(\eta_{1}\right)\right|+\left|\mathcal{P}^{\prime \prime}\left(\eta_{2}\right)\right|\right)\right. \\
&\left.\quad+\sum_{i=1}^{n}\left[\frac{\alpha-4 \mathcal{K}}{6(\alpha+2 \mathcal{K})}-s^{i}\left(\frac{1}{3(i+1)(i+2)}-\frac{\Gamma\left(\frac{\alpha}{\mathcal{K}}+2\right) \Gamma(i+1)}{\Gamma\left(\frac{\alpha}{\mathcal{K}}+i+3\right)}\right)\right]\left|\mathcal{P}^{\prime \prime}\left(\frac{\eta_{1}+\eta_{2}}{2}\right)\right|\right] .
\end{aligned}
$$

\section{Conclusion}

In this paper, we have introduced a new class of $n$-polynomial $s$-type convex functions, derived several new versions of the Hermite-Hadamard and Ostrowski type inequalities using the class of $n$-polynomial $s$-type convex functions, provided two integral identities for the first and second order differentiable functions, and obtained some refinements of the Ostrowski type inequality. We have also discussed some special cases for the obtained results which showed that the results obtained are quite unifying one. The outcomes acquired by the future plan are all the more invigorating as contrasted with results accessible in the literature. Finally, our work's consequences have a potential connection with fractal theory and machine learning [65-67].

\section{Acknowledgements}

The authors would like to express their sincere thanks to the editor and the anonymous reviewers for their helpful comments and suggestions.

\section{Funding}

The work was supported by the Natural Science Foundation of China (Grant Nos. 11971142, 61673169, 11871202, 11701176, 11626101, 11601485). 


\section{Competing interests}

The authors declare that they have no competing interests.

\section{Authors' contributions}

All authors contributed equally to the writing of this paper. All authors read and approved the final manuscript.

\section{Author details}

'Department of Mathematics, Government College University, Faisalabad, Pakistan. ${ }^{2}$ Department of Mathematics, Faculty of Arts and Sciences, Giresun University, Giresun, Turkey. ${ }^{3}$ Department of Mathematics, Faculty of Arts and Sciences, Çankaya University, Ankara, Turkey. ${ }^{4}$ Department of Mathematics, Huzhou University, Huzhou, China. ${ }^{5}$ Hunan Provincial Key Laboratory of Mathematical Modeling and Analysis in Engineering, Changsha University of Science \& Technology, Changsha, China.

\section{Publisher's Note}

Springer Nature remains neutral with regard to jurisdictional claims in published maps and institutional affiliations.

Received: 3 April 2020 Accepted: 24 May 2020 Published online: 03 June 2020

\section{References}

1. Wu, J., Liu, Y.-C.: Fixed point theorems for monotone operators and applications to nonlinear elliptic problems. Fixed Point Theory Appl. 2013, Article ID 134 (2013)

2. Wu, J.: Some fixed-point theorems for mixed monotone operators in partially ordered probabilistic metric spaces. Fixed Point Theory Appl. 2014, Article ID 49 (2014)

3. Huang, C.-X., Guo, S., Liu, L.-Z.: Boundedness on Morrey space for Toeplitz type operator associated to singular integral operator with variable Calderón-Zygmund kernel. J. Math. Inequal. 8(3), 453-464 (2014)

4. Zhou, X.-S.: Weighted sharp function estimate and boundedness for commutator associated with singular integral operator satisfying a variant of Hörmander's condition. J. Math. Inequal. 9(2), 587-596 (2015)

5. Huang, C.-X., Liu, L.-Z.: Boundedness of multilinear singular integral operator with a non-smooth kernel and mean oscillation. Quaest. Math. 40(3), 295-312 (2017)

6. Tan, Y.-X., Liu, L.-Z.: Weighted boundedness of multilinear operator associated to singular integral operator with variable Calderón-Zygmund kernel. Rev. R. Acad. Cienc. Exactas Fís. Nat., Ser. A Mat. 111(4), 931-946 (2017)

7. Hu, H.-J., Liu, L.-Z.: Weighted inequalities for a general commutator associated to a singular integral operator satisfying a variant of Hörmander's condition. Math. Notes 101(5-6), 830-840 (2017)

8. Rashid, S., Jarad, F., Noor, M.A., Kalsoom, H., Chu, Y.-M.: Inequalities by means of generalized proportional fractional integral operators with respect another function. Mathematics 7(12), Article ID 1225 (2019)

9. Kumar, D., Singh, J., Tanwar, K., Baleanu, D.: A new fractional exothermic reactions model having constant heat source in porous media with power, exponential and Mittag-Leffler laws. Int. J. Heat Mass Transf. 138, 1222-1227 (2019)

10. Kumar, D., Singh, J., Baleanu, D.: On the analysis of vibration equation involving a fractional derivative with Mittag-Leffler law. Math. Methods Appl. Sci. 43(1), 443-457 (2020)

11. Rashid, S., Jarad, F., Chu, Y.-M.: A note on reverse Minkowski inequality via generalized proportional fractional integral operator with respect to another function. Math. Probl. Eng. 2020, Article ID 7630260 (2020)

12. Gorenflo, R., Mainardi, F.: Fractional Calculus: Integral and Differential Equations of Fractional Order. Springer, Vienna (1997)

13. Podlubny, I.: Fractional Differential Equations. Academic Press, San Diego (1999)

14. Abdeljawad, T., Baleanu, D.: Monotonicity results for fractional difference operators with discrete exponential kernels. Adv. Differ. Equ. 2017, Article ID 78 (2017)

15. Abdeljawad, T., Baleanu, D.: On fractional derivatives with exponential kernel and their discrete versions. Rep. Math. Phys. 80(1), 11-27 (2017)

16. Huang, C.-X., Liu, L.-Z.: Sharp function inequalities and boundness for Toeplitz type operator related to general fractional singular integral operator. Publ. Inst. Math. 92(106), 165-167 (2012)

17. Wu, J., Liu, Y.-C.: Uniqueness results and convergence of successive approximations for fractional differential equations. Hacet. J. Math. Stat. 42(2), 149-158 (2013)

18. Zhou, X.-S., Huang, C.-X., Hu, H.-J., Liu, L.: Inequality estimates for the boundedness of multilinear singular and fractional integral operators. J. Inequal. Appl. 2013, Article ID 303 (2013)

19. Liu, F.-W., Feng, L.-B., Anh, V., Li, J.: Unstructured-mesh Galerkin finite element method for the two-dimensional multi-term time-space fractional Bloch-Torrey equations on irregular convex domains. Comput. Math. Appl. 78(5), $1637-1650$ (2019)

20. Jiang, Y.-J., Xu, X.-J.: A monotone finite volume method for time fractional Fokker-Planck equations. Sci. China Math. 62(4), 783-794 (2019)

21. Zhou, S.-H., Jiang, Y.-J.: Finite volume methods for $\mathrm{N}$-dimensional time fractional Fokker-Planck equations. Bull. Malays. Math. Sci. Soc. 42, 3167-3186 (2019)

22. Pratap, A., Raja, R., Cao, J.-D., Alzabut, J., Huang, C.-X.: Finite-time synchronization criterion of graph theory perspective fractional-order coupled discontinuous neural networks. Adv. Differ. Equ. 2020, Article ID 97 (2020)

23. Iqbal, A., Adil Khan, M., Ullah, S., Chu, Y.-M.: Some new Hermite-Hadamard-type inequalities associated with conformable fractional integrals and their applications. J. Funct. Spaces 2020, Article ID 9845407 (2020)

24. Rashid, S., Jarad, F., Kalsoom, H., Chu, Y.-M.: On Pólya-Szegö and Ćebyšev type inequalities via generalized k-fractional integrals. Adv. Differ. Equ. 2020, Article ID 125 (2020)

25. Awan, M.U., Talib, S., Chu, Y.-M., Noor, M.A., Noor, K.I.: Some new refinements of Hermite-Hadamard-type inequalities involving $\Psi_{k}$-Riemann-Liouville fractional integrals and applications. Math. Probl. Eng. 2020, Article ID 3051920 (2020) 
26. Wang, M.-K., Chu, Y.-M., Jiang, Y.-P.: Ramanujan's cubic transformation inequalities for zero-balanced hypergeometric functions. Rocky Mt. J. Math. 46(2), 679-691 (2016)

27. Xu, H.-Z., Chu, Y.-M., Qian, W.-M.: Sharp bounds for the Sándor-Yang means in terms of arithmetic and contra-harmonic means. J. Inequal. Appl. 2018, Article ID 127 (2018)

28. Adil Khan, M., Hanif, M., Khan, Z.A., Ahmad, K., Chu, Y.-M.: Association of Jensen's inequality for s-convex function with Csiszár divergence. J. Inequal. Appl. 2019, Article ID 162 (2019)

29. Qian, W.-M., He, Z.-Y., Zhang, H.-W., Chu, Y.-M.: Sharp bounds for Neuman means in terms of two-parameter contraharmonic and arithmetic mean. J. Inequal. Appl. 2019, Article ID 168 (2019)

30. Qian, W.-M., Yang, Y.-Y., Zhang, H.-W., Chu, Y.-M.: Optimal two-parameter geometric and arithmetic mean bounds for the Sándor-Yang mean. J. Inequal. Appl. 2019, Article ID 287 (2019)

31. Zaheer Ullah, S., Adil Khan, M., Chu, Y.-M.: A note on generalized convex functions. J. Inequal. Appl. 2019, Article ID $291(2019)$

32. Qian, W.-M., Zhang, W., Chu, Y.-M.: Bounding the convex combination of arithmetic and integral means in terms of one-parameter harmonic and geometric means. Miskolc Math. Notes 20(2), 1157-1166 (2019)

33. Wang, M.-K., He, Z.-Y., Chu, Y.-M.: Sharp power mean inequalities for the generalized elliptic integral of the first kind. Comput. Methods Funct. Theory 20(1), 111-124 (2020)

34. Wang, M.-K., Hong, M.-Y., Xu, Y.-F., Shen, Z.-H., Chu, Y.-M.: Inequalities for generalized trigonometric and hyperbolic functions with one parameter. J. Math. Inequal. 14(1), 1-21 (2020)

35. Zhao, T.-H., Shi, L., Chu, Y.-M.: Convexity and concavity of the modified Bessel functions of the first kind with respect to Hölder means. Rev. R. Acad. Cienc. Exactas Fís. Nat., Ser. A Mat. 114(2), Article ID 96 (2020) https://doi.org/10.1007/s13398-020-00825-3

36. Huang, C.-X., Yang, Z.-C., Yi, T.-S., Zou, X.-F.: On the basins of attraction for a class of delay differential equations with non-monotone bistable nonlinearities. J. Differ. Equ. 256(7), 2101-2114 (2014)

37. Wang, J.-F., Chen, X.-Y., Huang, L.-H.: The number and stability of limit cycles for planar piecewise linear systems of node-saddle type. J. Math. Anal. Appl. 469(1), 405-427 (2019)

38. Wang, J.-F., Huang, C.-X., Huang, L.-H.: Discontinuity-induced limit cycles in a general planar piecewise linear system of saddle-focus type. Nonlinear Anal. Hybrid Syst. 33, 162-178 (2019)

39. Hu, X.-M., Tian, J.-F., Chu, Y.-M., Lu, Y.-X.: On Cauchy-Schwarz inequality for N-tuple diamond-alpha integral. J. Inequal. Appl. 2020, Article ID 8 (2020)

40. Abbas Baloch, I., Chu, Y.-M.: Petrović-type inequalities for harmonic h-convex functions. J. Funct. Spaces 2020, Article ID 3075390 (2020)

41. Wang, B., Luo, C.-L., Li, S.-H., Chu, Y.-M.: Sharp one-parameter geometric and quadratic means bounds for the Sándor-Yang means. Rev. R. Acad. Cienc. Exactas Fís. Nat., Ser. A Mat. 114(1), Article ID 7 (2020). https://doi.org/10.1007/s13398-019-00734-0

42. Yang, Z.-H., Qian, W.-M., Zhang, W., Chu, Y.-M.: Notes on the complete elliptic integral of the first kind. Math. Inequal. Appl. 23(1), 77-93 (2020)

43. Qian, W.-M., He, Z.-Y., Chu, Y.-M.: Approximation for the complete elliptic integral of the first kind. Rev. R. Acad. Cienc. Exactas Fís. Nat., Ser. A Mat. 114(2), Article ID 57 (2020). https://doi.org/10.1007/s13398-020-00784-9

44. Rafeeq, S., Kalsoom, S., Hussain, S., Rashid, S., Chu, Y.-M.: Delay dynamic double integral inequalities on time scales with applications. Adv. Differ. Equ. 2020, Article ID 40 (2020)

45. Rashid, S., Ashraf, R., Noor, M.A., Noor, K.I., Chu, Y.-M.: New weighted generalizations for differentiable exponentially convex mapping with application. AIMS Math. 5(4), 3525-3546 (2020)

46. Huang, C.-X., Zhang, H., Huang, L.-H.: Almost periodicity analysis for a delayed Nicholson's blowflies model with nonlinear density-dependent mortality term. Commun. Pure Appl. Anal. 18(6), 3337-3349 (2019)

47. Zhang, J., Huang, C.-X.: Dynamics analysis on a class of delayed neural networks involving inertial terms. Adv. Differ. Equ. 2020, Article ID 120 (2020)

48. Huang, C.-X., Long, X., Huang, L.-H., Fu, S.: Stability of almost periodic Nicholson's blowflies model involving patch structure and mortality terms. Can. Math. Bull. 63(2), 405-422 (2020)

49. Chu, Y.-M., Adil Khan, M., Ali, T., Dragomir, S.S.: Inequalities for $\alpha$-fractional differentiable functions. J. Inequal. Appl. 2017, Article ID 93 (2017)

50. Budak, H., Usta, F., Sarikaya, M.Z.: New upper bounds of Ostrowski type integral inequalities utilizing Taylor expansion Hacet. J. Math. Stat. 47(3), 567-578 (2018)

51. Adil Khan, M., Iqbal, A., Suleman, M., Chu, Y.-M.: Hermite-Hadamard type inequalities for fractional integrals via Green's function. J. Inequal. Appl. 2018, Article ID 161 (2018)

52. Usta, F., Budak, H., Sarikaya, M.Z., Set, E.: On generalization of trapezoid type inequalities for s-convex functions with generalized fractional integral operators. Filomat 32(6), 2153-2171 (2018)

53. Budak, H., Usta, F., Sarikaya, M.Z.: Refinements of the Hermite-Hadamard inequality for co-ordinated convex mappings. J. Appl. Anal. 25(1), 73-81 (2019)

54. Budak, H., Usta, F., Sarikaya, M.Z., Ozdemir, M.E.: On generalization of midpoint type inequalities with generalized fractional integral operators. Rev. R. Acad. Cienc. Exactas Fís. Nat., Ser. A Mat. 113(2), 769-790 (2019)

55. Usta, F., Budak, H., Sarikaya, M.Z.: Montgomery identities and Ostrowski type inequalities for fractional integral operators. Rev. R. Acad. Cienc. Exactas Fís. Nat., Ser. A Mat. 113(2), 1059-1080 (2019)

56. Latif, M.A., Rashid, S., Dragomir, S.S., Chu, Y.-M.: Hermite-Hadamard type inequalities for co-ordinated convex and qausi-convex functions and their applications. J. Inequal. Appl. 2019, Article ID 317 (2019)

57. Rashid, S., Noor, M.A., Noor, K.I., Safdar, F., Chu, Y.-M.: Hermite-Hadamard type inequalities for the class of convex functions on time scale. Mathematics 7(10), Article ID 956 (2019)

58. Adil Khan, M., Mohammad, N., Nwaeze, E.R., Chu, Y.-M.: Quantum Hermite-Hadamard inequality by means of a Green function. Adv. Differ. Equ. 2020, Article ID 99 (2020)

59. Awan, M.U., Akhtar, N., Iftikhar, S., Noor, M.A., Chu, Y.-M.: New Hermite-Hadamard type inequalities for $n$-polynomial harmonically convex functions. J. Inequal. Appl. 2020, Article ID 125 (2020)

60. Khan, S., Adil Khan, M., Chu, Y.-M.: Converses of the Jensen inequality derived from the Green functions with applications in information theory. Math. Methods Appl. Sci. 43(5), 2577-2587 (2020) 
61. Hadamard, J.: Étude sur les propriétés des fonctions entières et en particulier d'une fonction considérée par Riemann. J. Math. Pures Appl. 58, 171-215 (1893)

62. Ostrowski, A.: Über die Absolutabweichung einer differentiierbaren Funktion von ihrem Integralmittelwert. Comment. Math. Helv. 10(1), 226-227 (1937)

63. Adil Khan, M., Chu, Y.-M., Khan, T.U., Khan, J.: Some new inequalities of Hermite-Hadamard type for s-convex functions with applications. Open Math. 15(1), 1414-1430 (2017)

64. Rashid, S., Noor, M.A., Noor, K.I., Chu, Y.-M.: Ostrowski type inequalities in the sense of generalized $\mathcal{K}$-fractional integral operator for exponentially convex functions. AIMS Math. 5(3), 2629-2645 (2020)

65. Kwun, Y.C., Shahid, A.A., Nazeer, W., Abbas, M., Kang, S.M.: Fractal generation via CR iteration scheme with s-convexity. IEEE Access 7, 69986-69997 (2019)

66. Kumari, S., Kumari, M., Chugh, R.: Generation of new fractals via SP orbit with s-convexity. Int. J. Eng. Sci. 9(3), 2491-2504 (2017)

67. Wang, W.-J., Zhang, G.-P., Yang, L.-M., Wang, W.: Research on garment pattern design based on fractal graphics. EURASIP J. Image Video Process. 2019, Article ID 29 (2019)

68. Dragomir, S.S., Pečarić, J., Persson, L.E.: Some inequalities of Hadamard type. Soochow J. Math. 21(3), 335-341 (1995)

69. Toplu, T., Kadakal, M., İscan, I.: On n-polynomial convexity and some related inequalities. AlMS Math. 5(2), 1304-1318 (2020)

70. Mubeen, S., Habibullah, G.M.: k-Fractional integrals and application. Int. J. Contemp. Math. Sci. 7(1-4), 89-94 (2012)

71. Díaz, R., Pariguan, E.: On hypergeometric functions and Pochhammer k-symbol. Divulg. Mat. 15(2), 179-192 (2007)

72. Zhao, T.-H., Chu, Y.-M., Wang, H.: Logarithmically complete monotonicity properties relating to the gamma function. Abstr. Appl. Anal. 2011, Article ID 896483 (2011)

73. Yang, Z.-H., Qian, W.-M., Chu, Y.-M., Zhang, W.: On rational bounds for the gamma function. J. Inequal. Appl. 2017, Article ID 210 (2017)

74. Farid, G., Usman, M.: Ostrowski type fractional integral inequalities for $s$-Godunova-Levin functions via $k$-fractional integrals. Proyecciones 36(4), 753-767 (2017)

75. Park, J.: On some integral inequalities for twice differentiable quasi-convex and convex functions via fractional integrals. Appl. Math. Sci. 9(62), 3057-3069 (2015)

\section{Submit your manuscript to a SpringerOpen ${ }^{\circ}$ journal and benefit from:}

- Convenient online submission

- Rigorous peer review

- Open access: articles freely available online

- High visibility within the field

- Retaining the copyright to your article

Submit your next manuscript at $\gg$ springeropen.com 